\title{
Divertor impurity seeding experiments at the COMPASS tokamak
}

\author{
M. Komm ${ }^{1}$, I. Khodunov ${ }^{1,2}$, J. Cavalier ${ }^{1}$, P. Vondracek ${ }^{1,3}$, S. Henderson ${ }^{4}$, \\ J. Seidl ${ }^{1}$, J. Horacek ${ }^{1}$, D. Naydenkova ${ }^{1}$, J. Adamek ${ }^{1}$, P. Bilkova ${ }^{1}$, P.

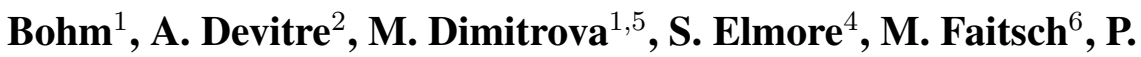 \\ Hacek $^{1,3}$, J. Havlicek ${ }^{1}$, A. Havranek ${ }^{1}$, M. Imrisek ${ }^{1,3}$, J. Krbec ${ }^{1}$, M.

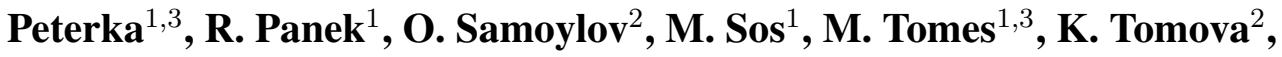 \\ V. Weinzettl ${ }^{1}$ and the EUROfusion MST1 team ${ }^{7}$ \\ ${ }^{1}$ Institute of Plasma Physics of the CAS, Za Slovankou 3, 18200 Prague 8, Czech Republic \\ ${ }^{2}$ Department of Applied Physics, Gent University, Gent, Belgium \\ ${ }^{3}$ Charles Univ, Fac Math \& Phys, V Holesovickach 2, 18000 Prague 8, Czech Republic \\ ${ }^{4}$ Culham Sci Ctr, CCFE, Abingdon OX14 3DB, Oxon, England \\ ${ }^{5}$ Emil Djakov Institute of Electronics, Bulgarian Academy of Sciences, 72, Tsarigradsko \\ Chaussee, 1784 Sofia, Bulgaria \\ ${ }^{6}$ Max-Planck-Institut fur Plasmaphysik, 85748 Garching b. Munchen, Germany \\ ${ }^{7}$ See the author list of Meyer H. et al 2017 Nucl. Fusion 57102014 \\ E-mail: kommeipp.cas.cz
}

\begin{abstract}
Partial detachment is the desired regime for the baseline burning plasma scenario in ITER and next-step devices, as it allows to dissipate the majority of the energy carried by charged particles through the scrape-off-layer (SOL) and thus avoids localised heat flux deposition in the divertor region. The COMPASS tokamak is equipped with an open divertor and has a relatively short connection length, both factors being unfavourable for access to detachment. As such, it only allows to approach naturally detached operation at very high lineaveraged densities $\left(>10^{20} \mathrm{~m}^{-3}\right.$ ), which are incompatible e.g. with maintaining the ELMy $\mathrm{H}$-mode regime. In order to achieve the detachment at lower densities, impurities (such as nitrogen) must be injected into the plasma in the divertor region.

A series of experiments with impurity injection in the range of $1-9 \times 10^{20}$ molecules per second at different locations in the divertor were performed with the aim to cool the plasma and influence the particle and heat transport onto the divertor targets and provoke partial detachment. Previously reported results [M. Komm et al, EPS 2017, P1.118] were largely extended by injection of nitrogen at the outer divertor target.

In order to analyze the divertor heat flux footprint in seeded plasmas, the buffered heat flux $q_{B}$ was introduced, with radial profile being approximated by an exponential decay. A new set of generic parameters - the peak heat flux $q_{\text {peak }}$, the fraction of power reaching the target $f_{d i v}$ and divertor footprint spreading factor $S_{f}$ was proposed to characterise divertor footprint under detached conditions.
\end{abstract}




\section{Introduction}

The power exhaust in the divertor represents one of the key challenges of contemporary fusion research, as the heat fluxes in machines with burning plasmas, such as ITER or DEMO, can easily exceed the material limits of the plasma-facing components (PFCs) and lead to their damage and reduced lifetime [14]. Most of the heat flux escaping from the last closed flux surface (LCFS) is carried along the field lines until it reaches the PFCs in the divertor. To overcome the issue of divertor overheating in ITER, the partially detached regime is envisaged in the baseline scenario for burning plasmas [15]. Detachment allows to convert a majority of the energy carried by charged particles into radiation and as such prevents localised deposition of the heat fluxes [16]. It is characterised by an electron temperature gradient along the field lines, as they pass from the scrape-off layer (upstream) to the divertor target (downstream) and significant power loss in the SOL. The temperature at the target is typically low $(<5 \mathrm{eV})$, allowing a significant population of neutrals to form in the divertor region.

In general, there are two ways of achieving the detached regime - (i) by increasing the density until sufficient amount of collisions with charged and neutral particles leads to power dissipation and cooling of the downstream plasma, or (ii) by injection of selected impurities, often strong radiators, which also allows to remove the power by radiation. In this work, we will focus on the latter approach, and we will present results of impurity seeding experiments at the COMPASS tokamak, where access to detached operation is particularly difficult due to open divertor geometry and relatively short connection length. On the other hand, COMPASS has a ITER-like plasma shape and as such it can contribute to improve or formulate the relevant multi-machine scalings.

The physics of detachment is incredibly complex, including atomic physics, surface geometries and plasma-wall interaction. The aim of this contribution is to characterise the practical aspects of detachment, such as the reduction of plasma pressure and heat fluxes in the divertor. In order to do this, we introduce a novel set of generic parameters: peak divertor heat flux $q_{\text {peak }}$, fraction of power reaching the divertor $f_{d i v}$ and divertor footprint spreading factor $S_{f}$, which are able to describe important properties of virtually any profile, independently of the underlying physics processes.

\section{Experimental setup}

\subsection{Injection scenario}

The impurity was injected in a series of otherwise identical attached ohmically heated low confinement mode discharges $\left(I_{p}=-210 \mathrm{kA}, B_{T}=-1.38 \mathrm{~T}, n_{e}=5 \times 10^{19} \mathrm{~m}^{-3}\right)$, as shown in Fig. 1. After a series of comparative experiments with nitrogen and neon seeding, the nitrogen was selected as a more favourable impurity (allowing for a range of effects in the divertor without the risk of disruptions) and so all the experiments in this work refer to discharges with nitrogen seeding. The impurity particle flux was controlled by a pre-set waveform on a piezoelectric valve in the range of $1-9 \times 10^{20}$ molecules per second. A typical waveform included a $10 \mathrm{~ms}$ pre-puff during which the valve was requested to be fully opened (to ensure that the valve 


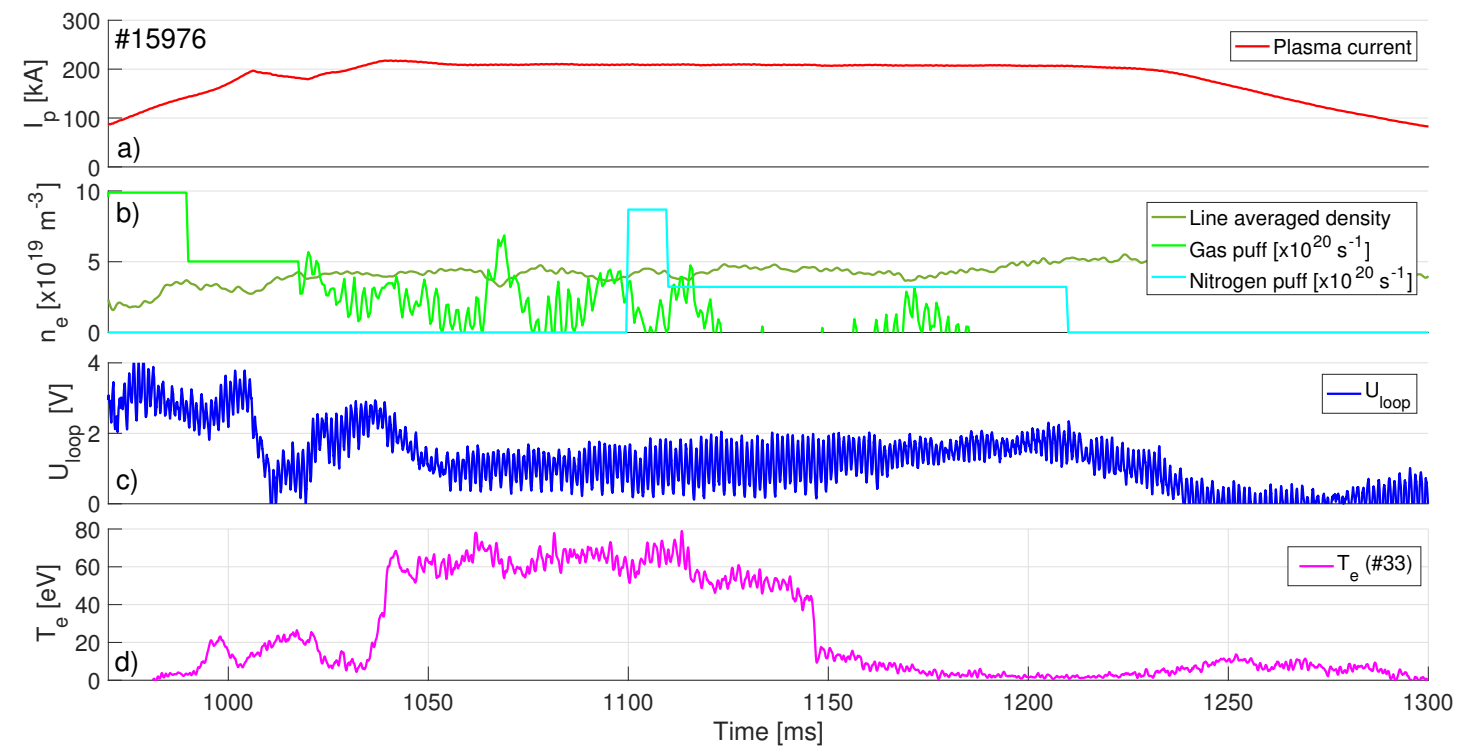

Figure 1. Overview of discharge \#19576 with nitrogen injected at the LFS target with profile of plasma current (A), line averaged density (B), loop voltage (C) and electron temperature recorded by probe set \#33 $(\mathrm{R}=490.6 \mathrm{~mm})$ at the LFS target.

mechanism starts to function properly), followed by a $100 \mathrm{~ms}$ constant puff at the desired particle flux (see Fig. 1B), which was varied on a shot-to-shot basis. Two different seeding locations were used: at first nitrogen injection at the inner target (major radius $\mathrm{R}=469 \mathrm{~mm}$ ) [1], later at the outer target $(\mathrm{R}=500 \mathrm{~mm})$, located outside the outer strike point. An example of electron temperature $T_{e}$ time evolution from probe set \#33 (located at $\mathrm{R}=490.6 \mathrm{~mm}$, meaning $10 \mathrm{~mm}$ outside the outer strike point) is shown in Fig. 1D. For reference in the future sections, we include the list of performed discharges in the summary table 1.

Table 1. List of discharges performed with LFS anf HFS nitrogen seeding HFS seeding LFS seeding

\begin{tabular}{|c|c|c|c|}
\hline Discharge & $\Gamma_{N_{2}}$ & Dischagre & $\Gamma_{N_{2}}$ \\
\hline- & & $\# 15972$ & $1.4 \times 10^{20} \mathrm{~s}^{-1}$ \\
\hline$\# 13729$ & $2.0 \times 10^{20} \mathrm{~s}^{-1}$ & $\# 15973$ & $2.0 \times 10^{20} \mathrm{~s}^{-1}$ \\
\hline$\# 13725$ & $2.8 \times 10^{20} \mathrm{~s}^{-1}$ & $\# 15975$ & $2.6 \times 10^{20} \mathrm{~s}^{-1}$ \\
\hline$\# 13730$ & $3.7 \times 10^{20} \mathrm{~s}^{-1}$ & $\# 15976$ & $3.2 \times 10^{20} \mathrm{~s}^{-1}$ \\
\hline$\# 13731$ & $4.5 \times 10^{20} \mathrm{~s}^{-1}$ & $\# 15977$ & $4.4 \times 10^{20} \mathrm{~s}^{-1}$ \\
\hline
\end{tabular}

\subsection{Influence of nitrogen content on probe measurements}

The divertor probes are the principal diagnostics for detachment studies at COMPASS. In the following analysis, we employ the combined array of 55 Ball-pen and 110 Langmuir probes (LP) (in two linear arrays), which allows for fast $(\sim \mu \mathrm{s})$ measurements of electron temperature $T_{e}$, ion saturation current $I_{\text {sat }}$ and floating potential $V_{\text {float }}$ [2]. This technique 

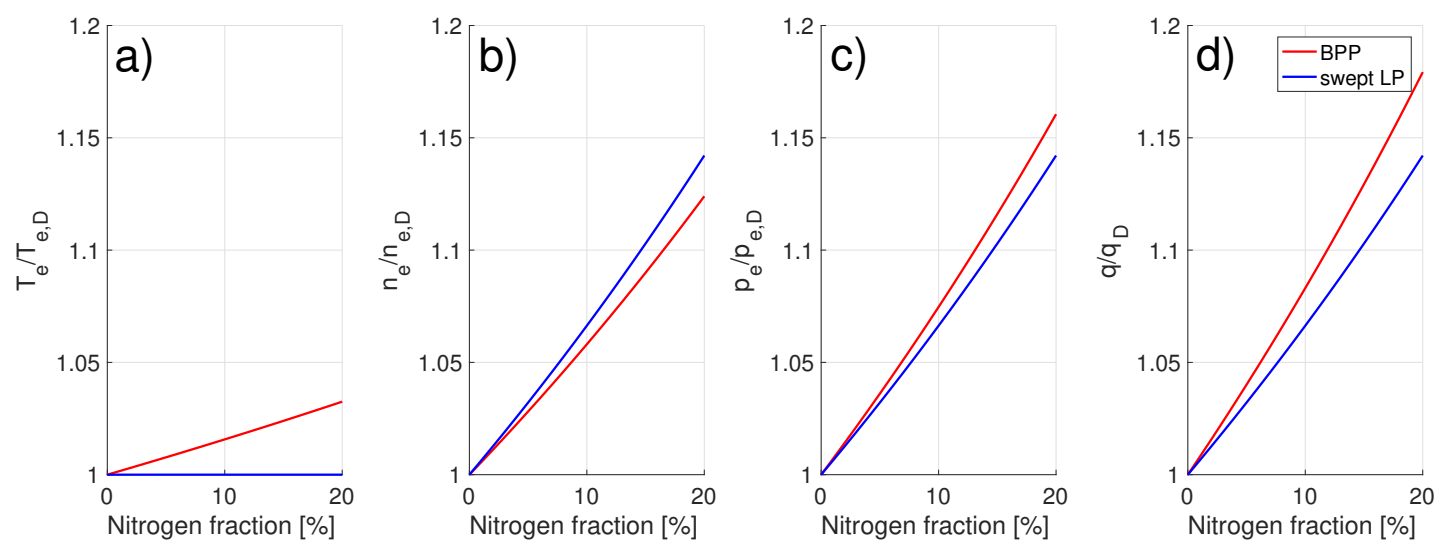

Figure 2. Imprecission of measurement of $\mathrm{T}_{e}(\mathrm{~A}), \mathrm{n}_{e}(\mathrm{~B})$ and $\mathrm{p}_{e}(\mathrm{C})$ for Ball-pen + Langmuir probes due to presence of nitrogen in the plasma. All values are normalized to measurements in pure deuterium.

has been validated in upstream measurements against Thomson scattering in COMPASS and AUG [3] and in selected cases also against the swept Langmuir probes of the same divertor probe array [2]. More recently, a comparison with measurements of the divertor infra-red thermography system [44] was also performed (in attached plasma conditions), yielding good agreement. However, a comparison with the array of 39 divertor swept Langmuir probes located at different toroidal location [46] did not bring satisfactory agreement, especially in terms of $T_{e}$. This disagreement is a subject of an ongoing research, however clearly outside the scope of this work. Note that $T_{e}$ measurements of the probe array in far SOL or during nitrogen seeding, when $T_{e}$ is low (below $5 \mathrm{eV}$ ), could not be validated against other diagnostics in COMPASS due to their insufficient operating range - infrared cameras have in general difficulties resolving very low heat fluxes [4] and swept probes are known to overestimate $T_{e}$ in such conditions[5][6]. However, it has been shown that BPP retains it operating properties even at low $T_{e}$ (see Fig. 4 in [3]).

The analysis of probe measurements in tokamaks is usually based on an assumption of plasma consisting of single ion species. This can be clearly violated in case there's intensive injection of nitrogen in deuterium plasmas. Unfortunately, it was not possible to determine the fraction of nitrogen ions at the location of probe measurements. However, we can model the effect of variable fraction of nitrogen in plasma and hence estimate the error which can be made by neglecting the presence of nitrogen in the analysis. In order to do that, we assume that the nitrogen is only singly ionized (which corresponds to the most probable state of nitrogen in the range of temperatures measured during the seeding phase) and we introduce the nitrogen density fraction $f_{N}=n_{N} / n_{i}$. We further assume that the only effect of nitrogen is a presence of ion current carried by nitrogen ions to the probes, omitting possible effects on ion collecting area, collisional effects in the sheath etc.

The temperature measurements are achieved using a combination of floating potentials 
of the Ball-pen and a Langmuir probes (LP), with the knowledge of a coefficient $\alpha_{L P}$ [2]. The value of the LP floating potential is influenced by the presence of nitrogen ions and scales as

$$
V_{f l} \sim \ln \left(\left(1-f_{N}\right) \sqrt{m_{D} / m_{e}}+f \sqrt{m_{N} / m_{e}}\right) / \ln \left(\sqrt{m_{D} / m_{e}}\right),
$$

where $m_{D}$ and $m_{N}$ are deuterium and nitrogen mass respectively. In absence of working theoretical model for the Ball-pen probe, we assume that coefficient $\alpha_{B P P}$ scales equally. This means that the total coefficient $\alpha=\alpha_{L P}-\alpha_{B P P}$ increases with increasing amount of nitrogen in the plasma. Since the dependence is logarithmic, only small variations of $T_{e}$ occur within the range of expected nitrogen fractions $\left(f_{N}<20 \%\right)$ as shown in Fig. 2. The classical method of electron temperature extraction from swept Langmuir probe is not affected, as it operates with a ratio $I(V) / I_{\text {sat }}$, where $I_{\text {sat }}$ is also determined experimentally and so it reflects the plasma conditions correctly.

A similar effect occurs when density is extracted from the measurements of ion saturation current. In this case the effective mass influences the ion sound speed and so the density scales as $\sim 1 /\left(1-f+f \sqrt{m_{D} / m_{N}}\right)$ (shown in Fig. 2B).

Finally, when these two influences are combined, the resulting error in electron pressure and heat flux measurement is shown in Figs. 2C and 2D respectively. Deviations from optimal measurements are observed, however the scale of introduced error is acceptable even for relatively large $(\sim 20 \%)$ concentrations of nitrogen for the technique employed in further analysis, as well as for the classical method, shown here for reference.

\subsection{Upstream measurements}

With respect to the upstream measurements, the major source of error is the determination of the location of the LCFS. Since the low-density plasmas in COMPASS are in the sheathlimited regime, it is not possible to determine the separatrix temperature from power balance as in larger machines [10]. Instead, the location of LCFS is determined from equilibrium reconstruction code EFIT, which is known to have limited precision. We will assume that the possible imprecision of the separatrix location is of systematic nature (at least in the range of studied discharges)[18] and we will focus on relative changes of upstream quantities.

\section{Effects of the seeding}

\subsection{Radiation measured by the visible cameras}

The location of nitrogen injection is in the field of view of the RIS system[8] - a pair of fast color cameras operating in the visible range. RIS1 camera was oriented with tangential field of view of the plasma and operated at $8 \mathrm{kHz}$ sampling frequency with resolution $1280 \times 624$ RGB pixels, while the RIS2 was providing wide angle radial view at $5 \mathrm{kHz}$ and resolution of $1280 \times 1000$ pixels. It was possible to identify 3 different patterns of radiation during nitrogen injection, which are shown in Figs. 3 and 4. The piezoelectric valve was requested to open at $\mathrm{t}=1100 \mathrm{~ms}$ and after approximately $15 \mathrm{~ms}$ it was possible to detect a new source of radiation around the injection location (Fig. 3A). Later, this radiation became more toroidally uniform 

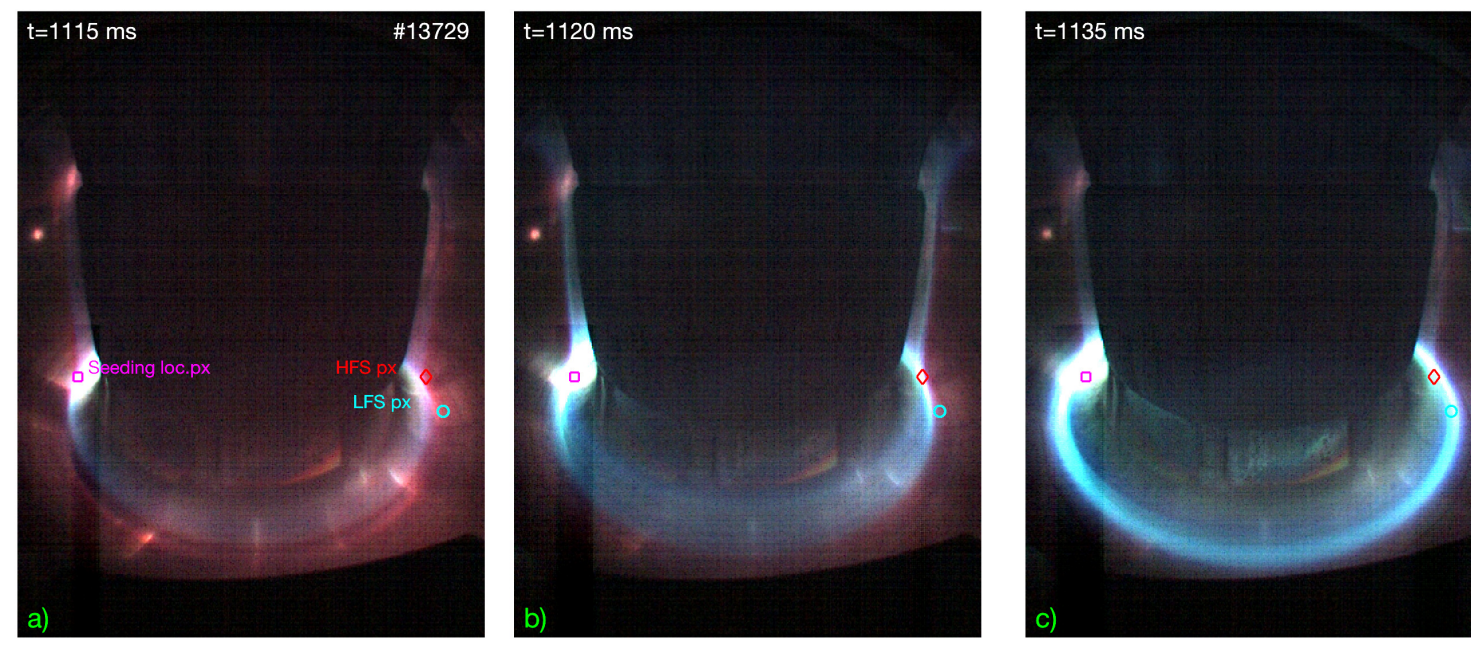

Figure 3. Three different patterns of nitrogen radiation in discharge $\# 13729\left(\Gamma_{N_{2}}=2.0 \times\right.$ $10^{20} \mathrm{~m}^{-3}$ ) observed by RIS1 camera with marked locations of seeding, LFS and HFS pixels.

but was restricted to the HFS (Fig. 3B), even in the cases when the injection was located at the outer strike point. In addition, measurements by the second RIS camera (see Fig. 4) shows that the radiation is not limited to the inner target but extends up to the whole HFS SOL. In some discharges, where the amount of injected nitrogen was sufficient, there was an abrupt change of the radiation pattern and the radiation became centred around the $\mathrm{x}$ point region (Fig. 3C). This transition was accompanied by a sudden change of temperature measured by the divertor probes, as shown in Fig. 5, for the discharge \#13729 (HFS seeding $\Gamma_{N_{2}}=2.0 \times 10^{20} \mathrm{~s}^{-1}$ ), where several such transitions were observed, since the amount of injected nitrogen was probably marginal with respect to detachment access. The pixel signal intensities (the total intensity from RGB channels was used) indeed follow this behaviour (patterns are labeled HFS and LFS in the figure) and at some times exhibit low-frequency oscillations (f $\sim 1 \mathrm{kHz}$ ), which according to RIS data are axisymmetric and ressemble those measured at AUG[30]. For the definition of $T_{e}$ plotted in Fig. 5 see section 3.4.

\subsection{Spectroscopy measurements}

The radiation of nitrogen was measured using a set of mini-spectrometers for near UV (247$473 \mathrm{~nm})$, visible $(460-663 \mathrm{~nm})$, and infra-red $(630-680 \mathrm{~nm})$ ranges with resolution of 0.15 , 0.17 , and $0.23 \mathrm{~nm}$, respectively [25]. The field of view covers the edge plasma at the outer midplane but excludes the outer target, as shown in 6B. It was possible to identify several nitrogen lines in the measured spectrum (see 6A). The most intensive line measured in the near UV to blue visible spectrum was the N IV multiplet lines near $348 \mathrm{~nm}$, with much weaker contributions from the N III multiplet lines near $410 \mathrm{~nm}$ and the N II singlet line at $399.5 \mathrm{~nm}$. The qualitative behaviour of the N IV multiplet intensity follows the increase of radiated fraction $f_{\text {rad }}=P_{\text {rad }} / P_{\text {ohm }}$ (where $P_{\text {rad }}$ is the total radiated power measured by bolometry[7] and $P_{\text {ohm }}$ is the ohmic input power), as shown in Fig. 7A and Fig. 7B.

We use the line-of-sight in the axis of the field of view cone and select the time slice near 

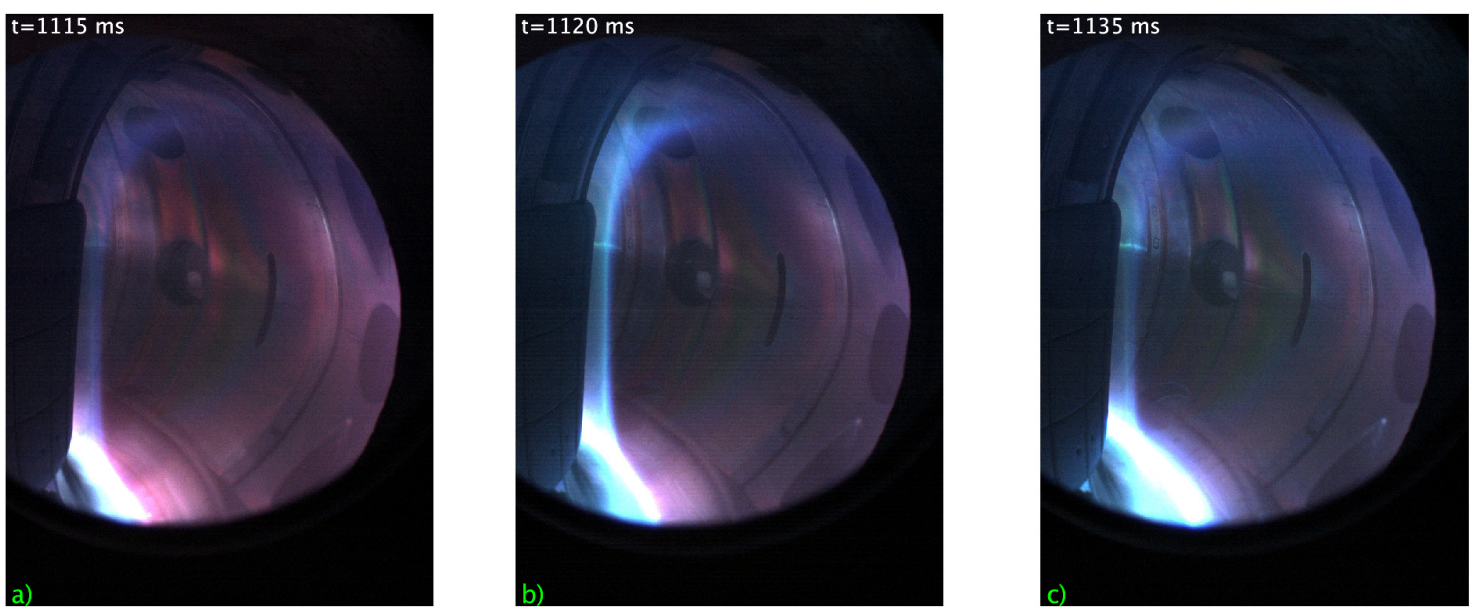

Figure 4. Three different patterns of nitrogen radiation in discharge \#13729 observed by the RIS2 camera.
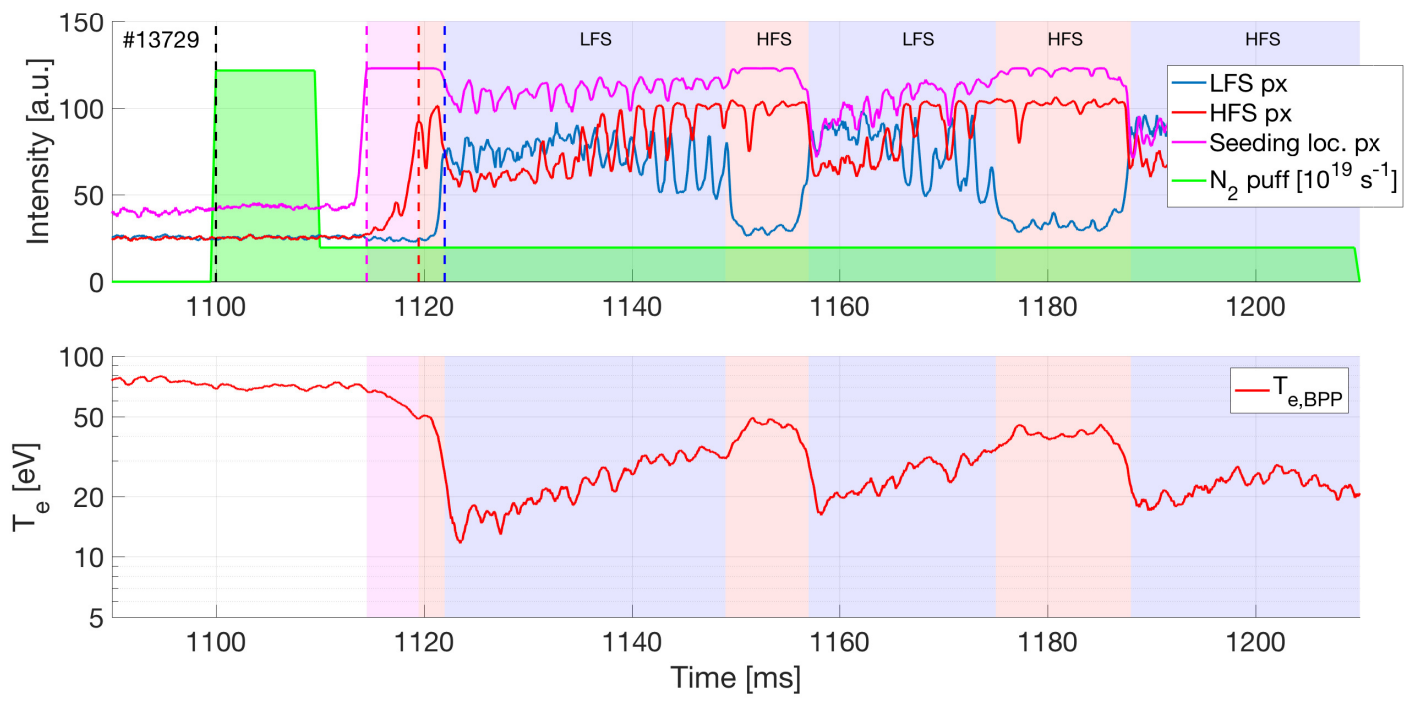

Figure 5. Pixel intensity at seeding location, HFS and LFS (top) and evolution of $T_{e}$ at LFS target (bottom). Background colors and labels indicate radiation regimes shown in Figs.3 and 4.

the beginning of the $\mathrm{N}_{2}$ seeding window ( $\mathrm{t}=1150 \mathrm{~ms}$ ) in shot \#15976 to discuss the model and necessary assumptions for estimating the $\mathrm{N}$ concentration based on the $\mathrm{N}$ IV multiplet intensity. The $\mathrm{N}$ concentration, $\mathrm{c}_{N}$, can be calculated using:

$$
c_{N}=\frac{I_{N I V}}{f_{N 3+} P E C^{e x c}+f_{N 4+} P E C^{r e c}} \frac{1}{\Delta L n_{e}^{2}}
$$

where $I_{N I V}$ is the N IV multiplet intensity, $f_{N 3+, 4+}$ are the ion fractional abundances of the $\mathrm{Z}=3+, 4+\mathrm{N}$ ions, $P E C^{e x c, r e c}$ are the photon emissivity rate coefficients for excitation and recombination, and $\Delta L$ is the length of the $\mathrm{N}$ IV emitting region through the line-of- 


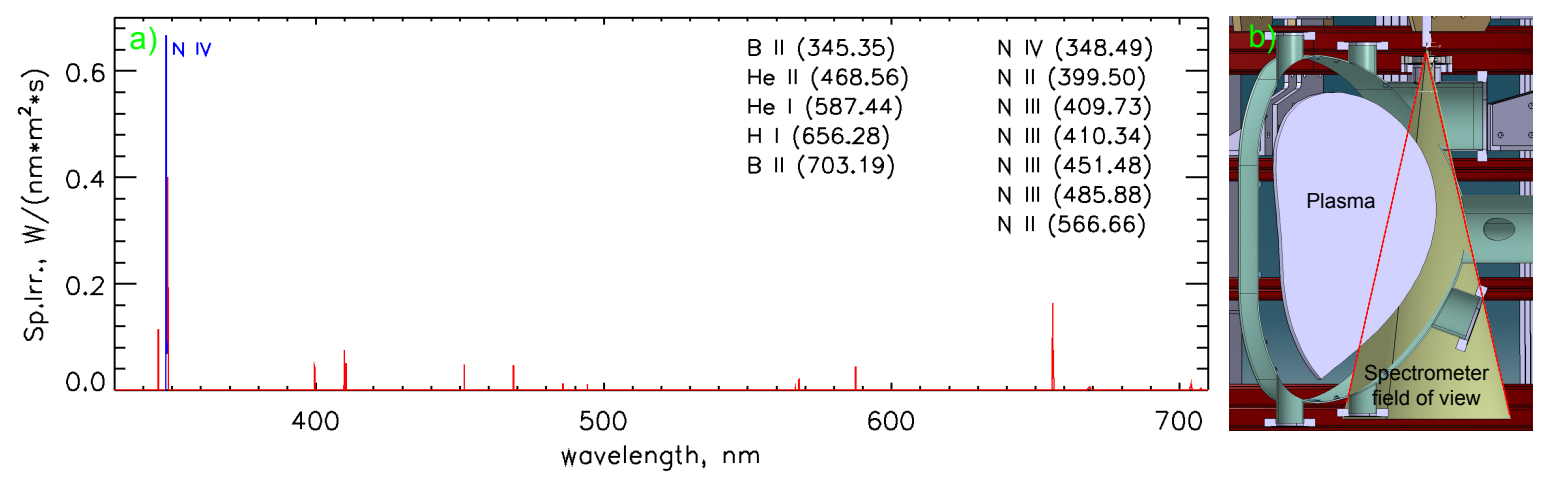

Figure 6. Nitrogen lines identified in the measured spectrum in discharge \#15976 at $\mathrm{t}=1150$ ms (a) and the field of view of the minispectrometers (b).
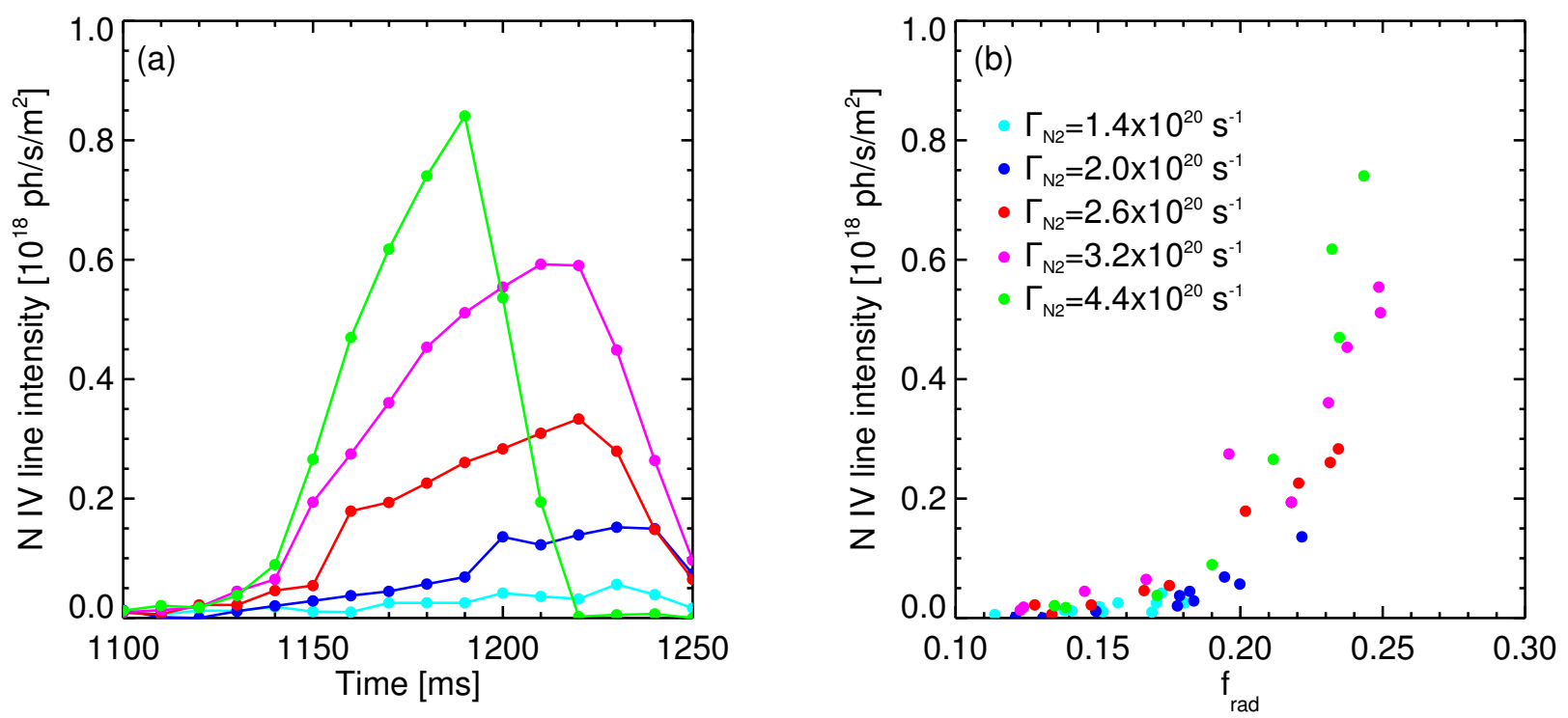

Figure 7. The intensity of the N IV multiplet line at $348 \mathrm{~nm}$ evolving in time (a) and as a function of radiated fraction $f_{\text {rad }}(\mathrm{b})$.

sight, which is approximated by the distance over which the line-of-sight penetrates inside the separatrix .

Firstly, to calculate the N IV multiplet intensity, a 4-Gaussian fit of the N IV multiplet emission feature and a single Gaussian fit of the nearby B II line is carried out as shown in Fig. 8A. Next, the ADAS excitation and recombination rates for this transition are interpolated as a function of temperature in Fig. 8B. From HRTS measurements at this particular time slice and shot, the separatrix density is $n_{e, s e p} \sim 2 \times 10^{19} \mathrm{~m}^{-3}$, while the temperature is $T_{e, s e p} \sim 40 \mathrm{eV}$. The fractional abundance of the $\mathrm{N}^{3+, 4+}$ charge states are then calculated using an ionisation balance with a transport loss term, $n_{e} \tau$, where $\tau$ is the impurity residence time in the separatrix. This parameter is not well known in the separatrix, or indeed anywhere in the SOL; however, one could begin by estimating it using the typical particle confinement times which range from $5-15 \mathrm{~ms}$ in COMPASS. In theory, this parameter could also account for enhanced recombination due to neutral deuterium atoms in the separatrix. For this study, the 

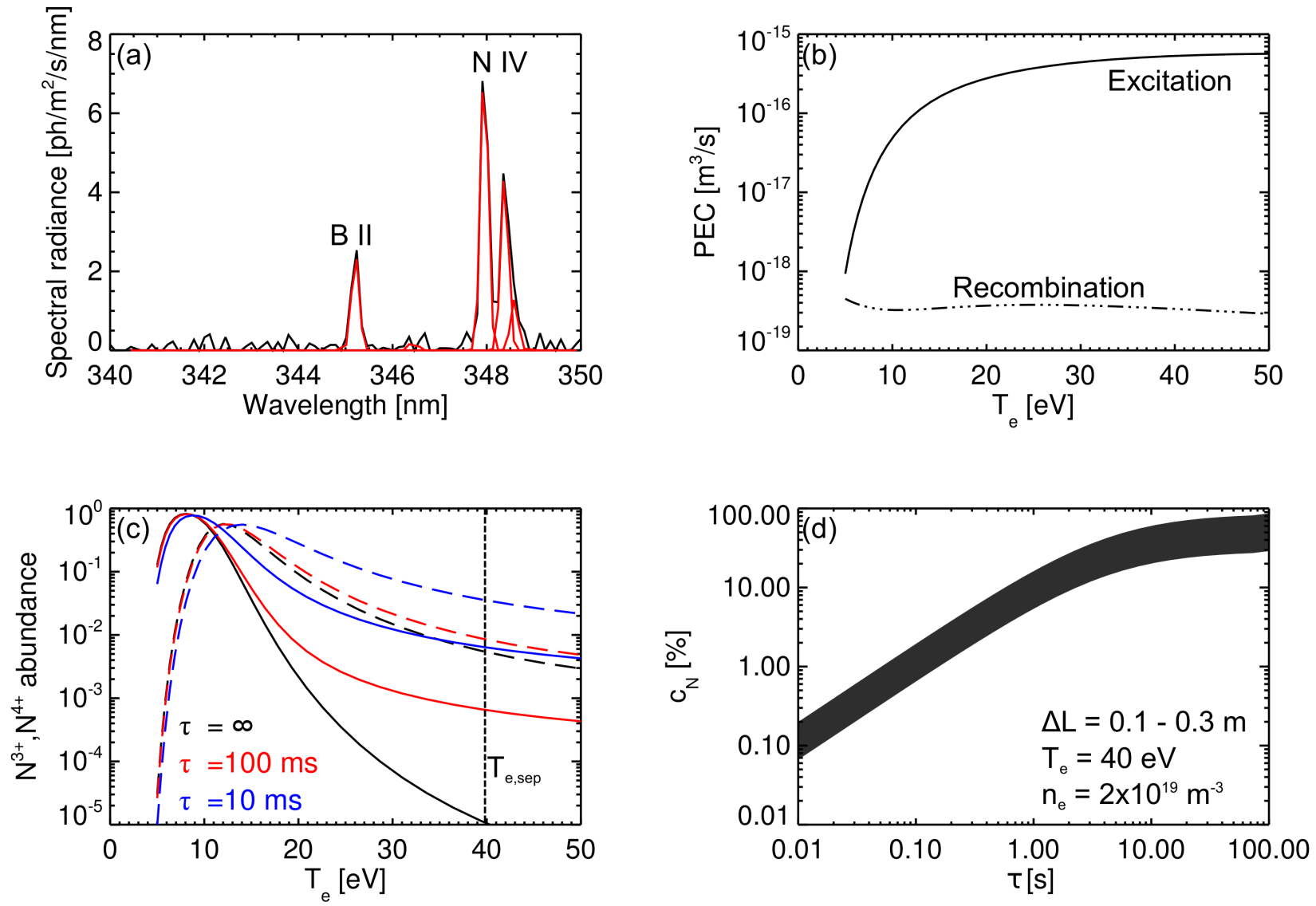

Figure 8. The measured spectral radiance (black) with Gaussian fit components (red) measured in the near UV for shot \#15976 at $\mathrm{t}=1150 \mathrm{~ms}$ is shown in (a). Excitation and recombination rates for the N IV multiplet transition at $\lambda \sim 348 \mathrm{~nm}$ are shown as a function of $T_{e}$ for $n_{e}=2 \times 10^{19} \mathrm{~m}^{-3}$ in (b). The ion fractions of $\mathrm{N}^{3+}$ (solid) and $\mathrm{N}^{4+}$ (dashed) are shown in (c) for three different impurity residence times. The $\mathrm{N}$ concentration at $T_{e}=40 \mathrm{eV}$ is shown as a function of $\tau$ in $(\mathrm{d})$.

ion fractions for three $\tau$ values of 10, 100, $\infty \mathrm{ms}$ are shown in Fig. 8C by the red, blue, and black lines, where the solid and dashed lines represent the $\mathrm{N}^{3+}$ and $\mathrm{N}^{4+}$ charge states, respectively. The latter $\tau$ value represents the zero-transport case where only ionisation and recombination rate coefficients from ADAS are considered.

The $c_{N}$ calculated at $T_{e, s e p}=40 \mathrm{eV}$ is shown in Fig. 8D as a function of $\tau$. The shaded region shows the upper and lower limit of $\Delta \mathrm{L}=0.1-0.3 \mathrm{~m}$. The $c_{N}$ is unphysically high (close to $100 \%)$ in the zero-transport case $(\tau>10 \mathrm{~s})$ suggesting that transport must be included. Between $\tau=10-100 \mathrm{~ms}$, the $c_{N}$ varies from $0.1-1.0 \%$ in the separatrix, which is likely a lower limit on the divertor nitrogen concentration since the ratio of the nitrogen concentration in the divertor and core plasma volumes has been observed on AUG to reach values of 10 [32]. These first results therefore provide a first insight into the divertor $\mathrm{N}$ concentrations required for detachment on COMPASS, however additional spectrometer sightlines isolating the emission from the divertor volume (e.g. following the analysis of [33]) and core CX measurements of the nitrogen density would help to validate these estimations. 


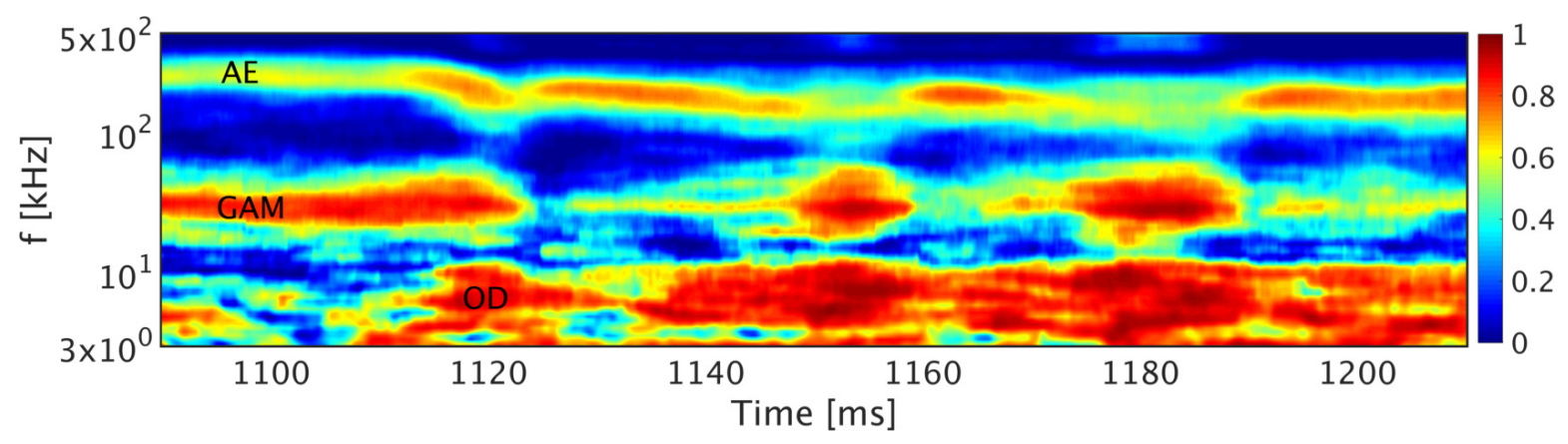

Figure 9. Wavelet cross-coherence between two Mirnov coils (MC17 and MA17), toroidally separated by $135^{\circ}$ and located in the HFS divertor region of COMPASS (\#13729).

\subsection{Magnetic measurements}

COMPASS is equipped with 2 sets of non-integrated Mirnov coils toroidally separated by $135^{\circ}$, each composed of 24 coils located in the same poloidal cross section (see Fig. 1 in [35]) that allow studies of the poloidal magnetic field generated by instabilities that take place during the discharge duration. In Fig. 9, we present the wavelet cross-coherence between the two toroidally separated Mirnov coils 17, located in the HFS divertor region of COMPASS, that shows how different instabilities during a nitrogen seeding experiment. One can observe that the MHD activity is strongly influenced by the nitrogen injection. Related to the first temperature drop ( $\sim 1120 \mathrm{~ms}$ ), a strong mode develops around $6 \mathrm{kHz}$ (labeled OD as onset of detachment) and grows back every time the transition between attached and detached plasma occurs ( 1155 and $1187 \mathrm{~ms}$ ). The geodesic acoustic mode [35] (labeled GAM around 30 $\mathrm{kHz}$ ) also strengthens during every onset of detachment while the Alvfen eigenmode [36][37] (labeled AE around $250 \mathrm{kHz}$ ) is damped. Between each divertor temperature drops, the GAM almost fully disappears while the AE mode builds up but with a lower frequency than prior to nitrogen seeding.

In nitrogen seeding experiments during $\mathrm{H}$-mode [38] and also in density ramp-up Lmode [39] on ASDEX Upgrade, strong fluctuations around $6 \mathrm{kHz}$ were observed by AXUV detectors near the x-point during the transition from attached to completely detached plasma. This phase called by the ASDEX team the fluctuating detachment state [40] may be linked to the development of the current convective instability [41]. As described in [41][42][43], such an instability develops when a large temperature difference exists between the inner and outer divertor, i.e. when the inner divertor detaches, as it usually detaches prior the outer one. Once the outer divertor detaches too, the temperature difference vanishes and the instability stabilises, so that the fluctuations disappear.

The mode visible around $6 \mathrm{kHz}$ in Fig. 10 seems to have a similar behaviour as the fluctuating detachment state described above since it appears mainly during the temperature drop in the divertor region. In addition, AXUV detectors looking near the X-point region also detect these fluctuations indicating that they take place at this location. Studies of the toroidal mode number with Mirnov coils show that the mode is toroidally symmetric. Last, the signals coming from the different poloidally separated Mirnov coils seem to indicate that 


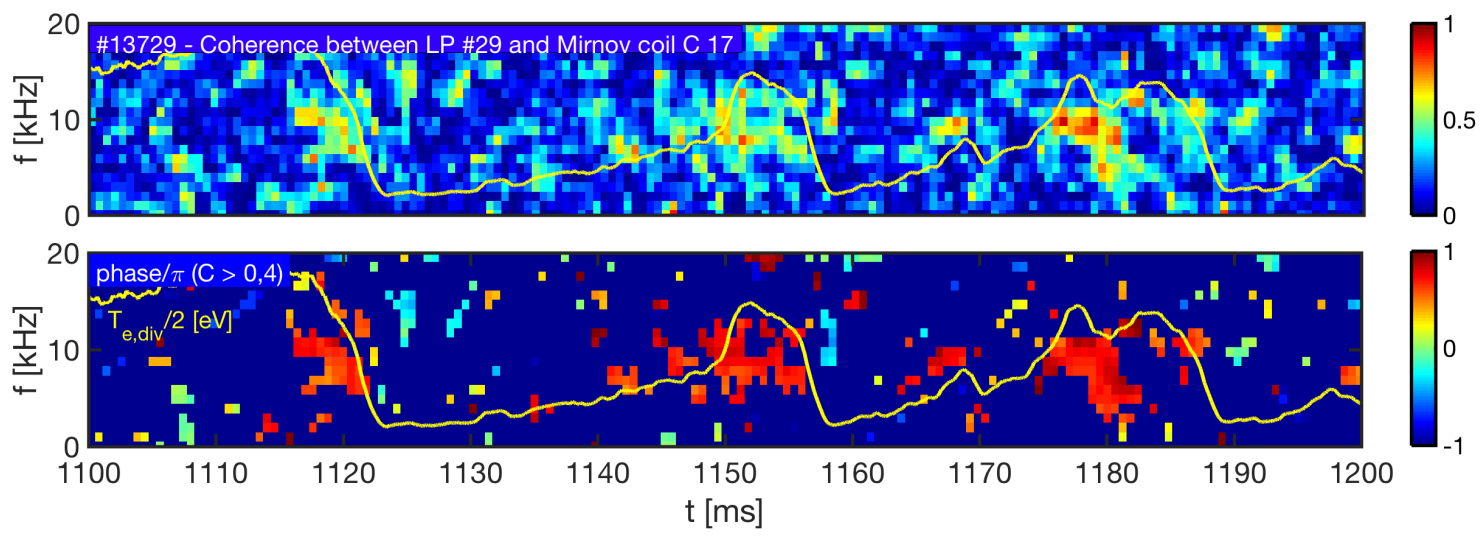

Figure 10. Coherence measurements: cross-coherence between $T_{e}$ measurement near the LFS strikepoint of \#13729 and the Mirnov coil MC17 (A) and associated phase divided by $\pi$ (B). Yellow line indicates $T_{e}$ measured by div. probes (divided by factor 2 ).

the instability is mostly present in the divertor region (coils 16-21) but also in the top part of the vessel (coils 5-10).

In Fig. 10, we show the coherence between the Mirnov coil MC17 and $T_{e}$ in the outer divertor measured by the combination of Ball-pen and Langmuir probes at $\mathrm{R}=483 \mathrm{~mm}$. The mode is strongly visible at the onset of detachment when the mean $T_{e}$ significantly drops. The fluctuations are also visible in the HFS divertor since the coherence between MC17 and a floating potential measurement near the HFS strike point shows a similar picture as shown in Fig. 9. The density, however, does not show any such fluctuations in both HFS and LFS indicating that the process is mainly linked to the temperature, supporting the physical picture described by [41].

\subsection{Effects in the divertor}

Figure 11 summarises the effects of nitrogen seeding on divertor temperature, pressure and heat flux, as measured by the combination of Langmuir and Ball-pen divertor probes. The values plotted in the figures correspond to the maximum quantities within $2 \mathrm{~cm}$ outside the OSP. The electron pressure was calculated as

$$
p_{e}=\left(1+M^{2}\right) n_{e} T_{e},
$$

where $M$ is the parallel Mach number, which was assumed to be equal to 1 at the target due to Bohm condition and equal to 0 upstream. The heat flux was calculated from the measured values of $T_{e}$ and $j_{\text {sat }}$. For simplicity it was assumed that the tiles were in ambipolar condition and so the parallel heat flux can be calculated as

$$
q_{\|}=\left(\gamma T e+E_{i}\right) j_{s a t},
$$

Where $E_{i}$ is the potential energy deposited by each ion-electron pair to the target, which is equal to a sum of ionisation energy (13.6 eV for deuterium) and surface bonding energy $(\sim 1 \mathrm{eV})$ [45]. The value of sheath heat transmission coefficient $\gamma$ depends on the ratio $T_{i} / T_{e}$ 

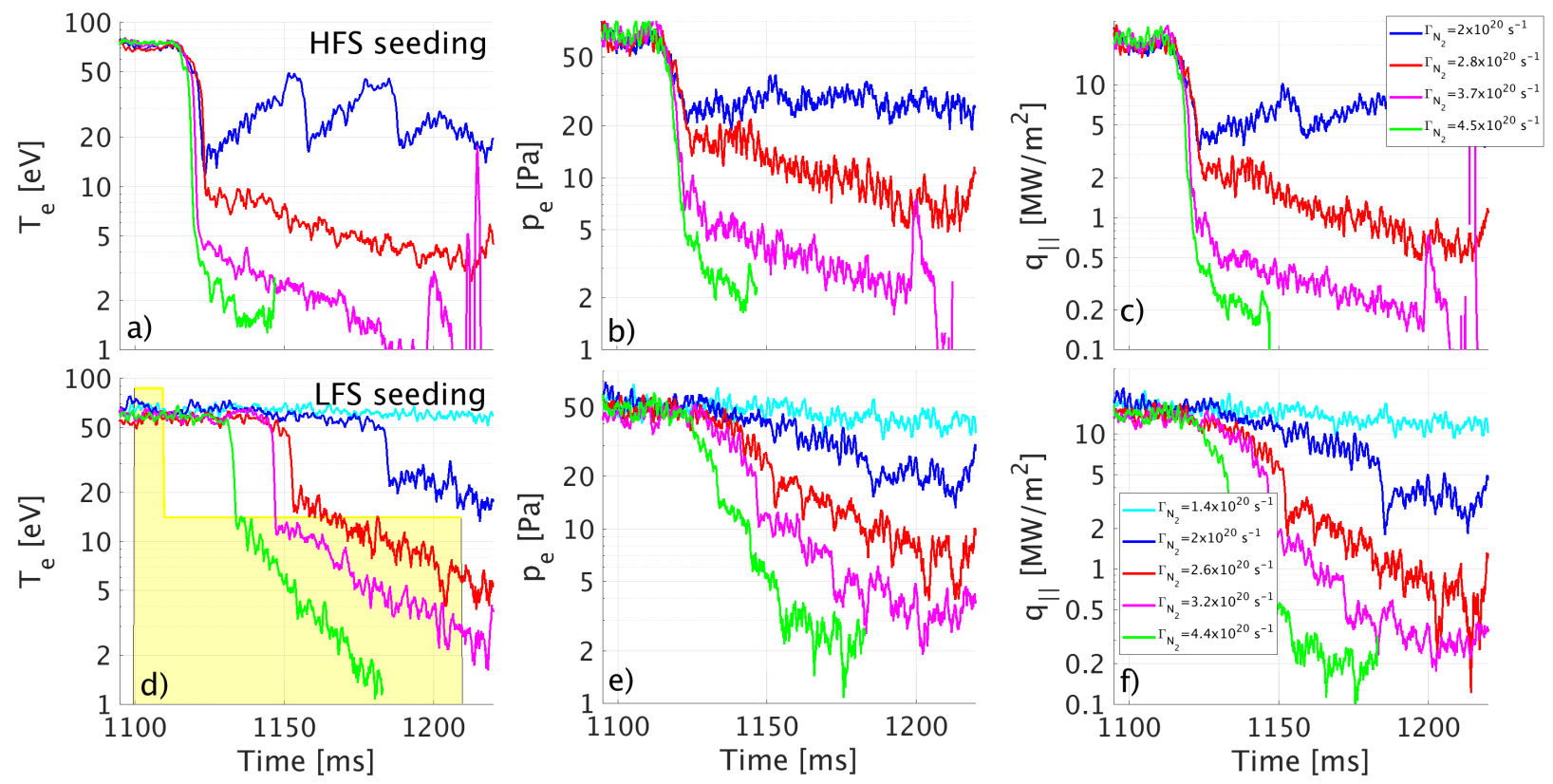

Figure 11. Temporal evolution of $T_{e}, p_{e}$ and $q_{||}$in the divertor for seeding at HFS (a-c) and LFS (d-f) measured by probes in the vicinity of the outer strike point.

and the coefficient of secondary emission [11]. We assume that the secondary emission is negligible due to the small angle of incidence of the field lines with respect to the tile top surface $\left(1-3^{\circ}\right)$ [12]. Following the recent comparison with divertor IR measurements [44], we adopt a value of $\gamma$ equal to 11 , which corresponds to $T_{i} / T_{e} \sim 2.5$. Note that our analysis concentrates on such properties of the heat flux profiles, which are not affected by a possibly different value of $\gamma$, as long as it is constant along the target.

The effects of the impurities are dependent on the location of the seeding. When the impurity was introduced at the HFS, it was always followed by an abrupt change of temperature (Fig. 11A) at the outer target, similar to behaviour which was observed at DIII$\mathrm{D}[20]$. For the highest amount of seeding, the discharge was ended prematurely due to a disruption caused by mode locking of a tearing mode.

When the nitrogen was seeded at the LFS, the effects in the divertor were generally more gradual (Fig. 11D-F). The abrupt response on the $T_{e}$ is still present but the relative magnitude of the drop is smaller and occurs at different times depending on the amount of nitrogen influx. At low seeding values there are no relaxations observed (unlike the case of HFS seeding, as shown in Fig. 11A-C), instead the target pressure and heat flux only gradually decrease. At highest amount of seeding there is still a disruption but it occurs only at the end of the flat-top phase of the discharge.

\subsection{Upstream and core parameters}

Due to the proximity of the seeding location to X-point and relatively low edge plasma temperatures in ohmic plasmas, it was expected that some of the injected nitrogen may 

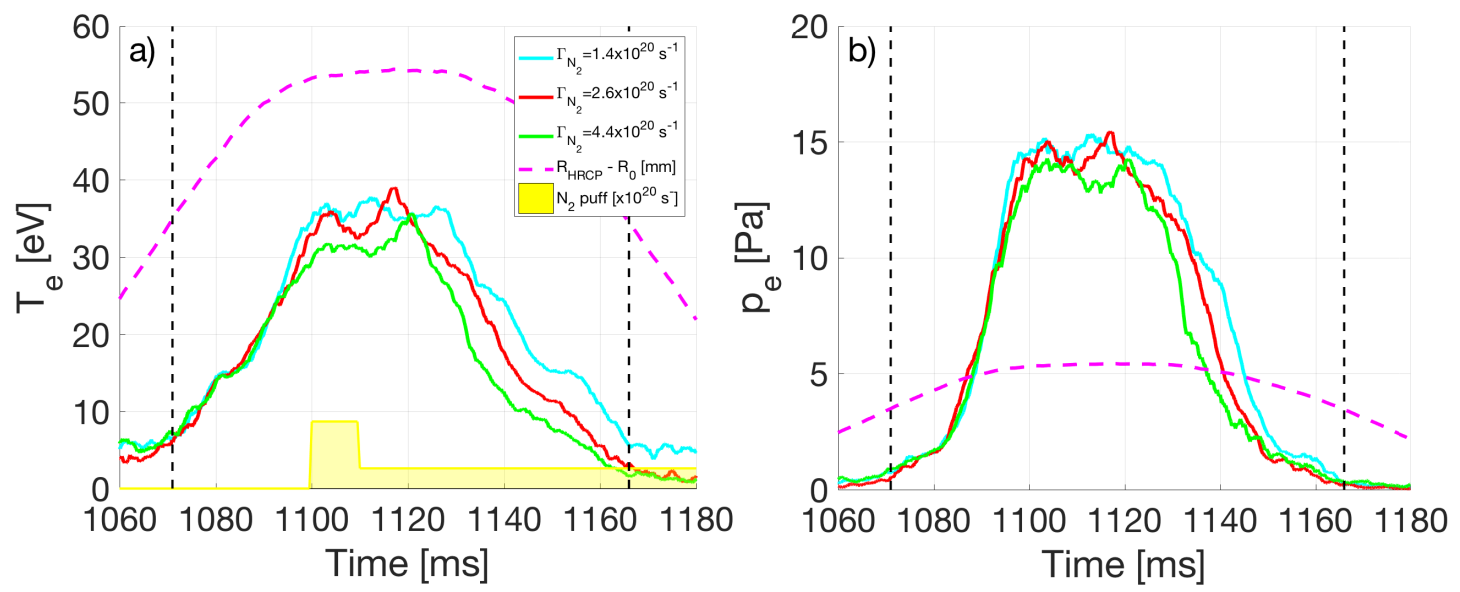

Figure 12. Time evolution of $T_{e}$ and $p_{e}$ during the movement of the HRCP. The vertical dashed lines indicate times where the probe penetrated beyond the limiter shadow.

penetrate into the confined plasma and cool it by radiation. As a consequence, a pressure drop would be observed on the upstream profile. This was confirmed by measurements performed by the horizontal reciprocating manipulator equipped with the probe head featuring a combination of Ball-pen and Langmuir probes[19]. The probe head was only allowed to probe far SOL to avoid possible perturbation of the divertor measurements. The position of LCFS according to the EFIT reconstruction was at $\mathrm{R}=0.728 \mathrm{~m}$, so the probe head was about $2 \mathrm{~cm}\left(\sim 2 \lambda_{p}\right)$ away from the LCFS during the deepest point of reciprocation. Note that the radial position of the probe $R_{h r c p}$ in Fig. 12 is plotted relative to the parking position $R_{0}=800$ $\mathrm{mm}$. The probe was moving during the discharges with seeding at LFS in such timing, that the inward motion was performed before the beginning of seeding, while the outward movement occurred during the seeding. Unfortunately the effect of nitrogen upstream was gradual over the duration of the movement (see e.g. Fig. 11D-F), and so it was not possible to extract a true radial profile of the measured quantities during the outward motion. However, the time evolution of $T_{e}$ and $p_{e}$ as shown in Fig. 12 demonstrate a reduction of both quantities during the outward motion, depending on the amount of injected nitrogen. The electron pressure was calculated as in Eq. 3. The density was deduced from the measurement of ion saturation current of a Langmuir pin assuming $T_{i} / T_{e}=2$ and the effective collecting area given by a recently developed model[17].

Another indication of the radiation in the confined plasma is provided by measurements

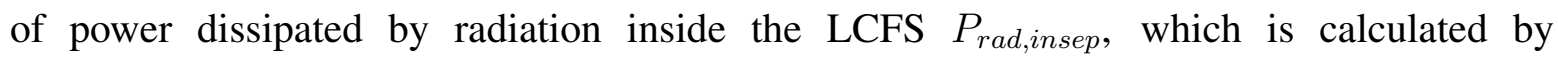
tomographic reconstruction of the AXUV bolometers measurements[7]. Also, measurements of the ohmic power $P_{o h m}$, obtained by magnetic measurement of $U_{\text {loop }}$, should show an increase due to higher $Z_{\text {eff }}$ caused by the presence of nitrogen. Both effects are well visible in the profiles presented in Fig. 13. Note that in \#15976 (magenta line in Fig. 13A), the initial radiation was significantly higher than in other discharges in the scan, however the relative change which occurred after the beginning of seeding is in line with trends observed in other discharges. 

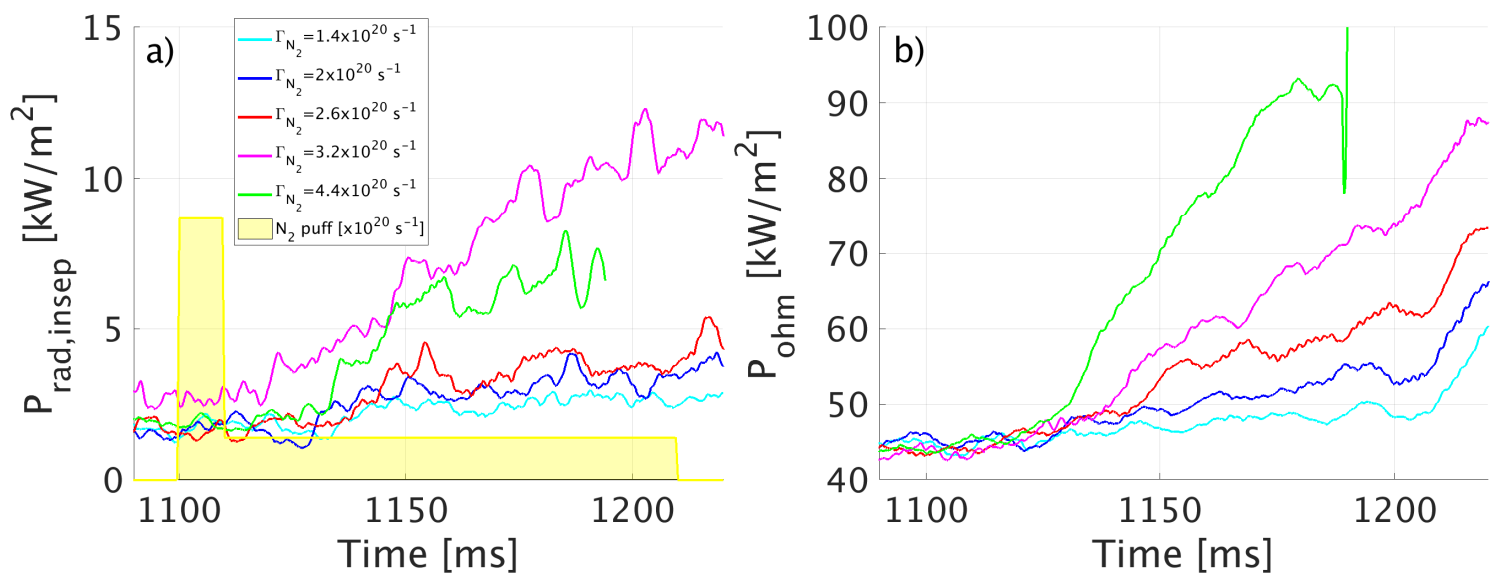

Figure 13. Time evolution of the plasma radiation measured by AXUV bolometers (left) and measurements of ohmic power $P_{\text {rad }}$.
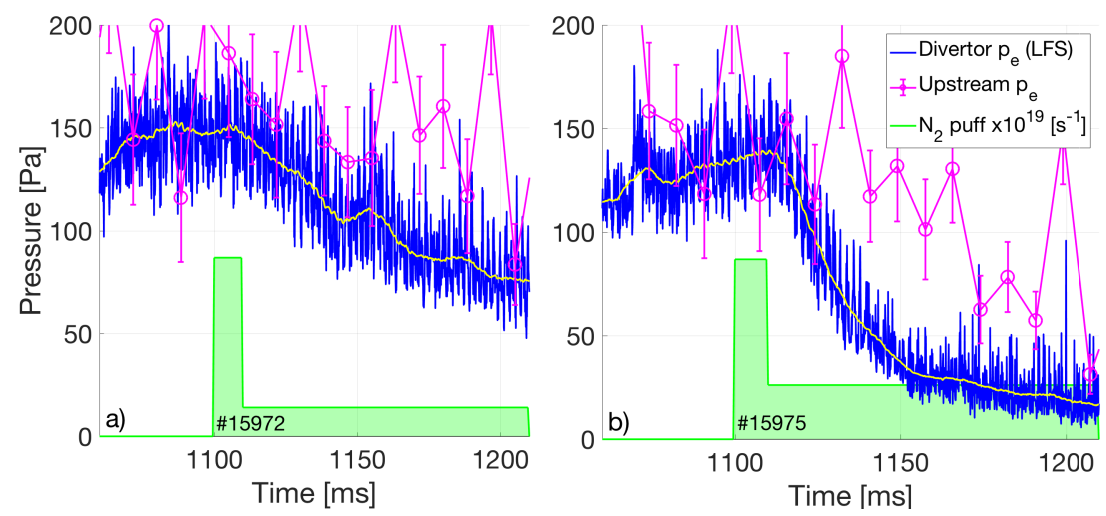

Figure 14. Evolution of upstream (magenta) and downstream (blue) pressure in discharges with low $\Gamma_{N_{2}}=1.4 \times 10^{20} s^{-1}$ (A) and high $\Gamma_{N_{2}}=3.2 \times 10^{20} s^{-1}$ (B) amount of seeding.

\subsection{Upstream and downstream pressure}

Findings presented during the previous section casted doubts whether the pronounced change in divertor parameters is due to nitrogen radiating in the SOL or whether the whole edge plasma is cooled. In order to distinguish between these two possibilities, the measurements of upstream and downstream pressures were compared. While the change of peak electron pressure at the outer target was measured using the LP+BPP divertor probes, the upstream pressure was monitored by HRTS[9] at the position of separatrix calculated by magnetic reconstruction. Both upstream and downstream pressures were calculated using Eq. 3.

It was observed that there is a significant drop of the downstream pressure following the injection of nitrogen and that the speed of the response and the magnitude of pressure drop can be controlled by the amount of injected nitrogen, as shown in Fig. 14. As expected, the effect of nitrogen was not restricted to the divertor only but also affected the upstream pressure. When this pressure drop (visible e.g. in Fig. 14B) was taken into account, it became difficult to determine whether the effect of nitrogen in the SOL is more significant than in the 


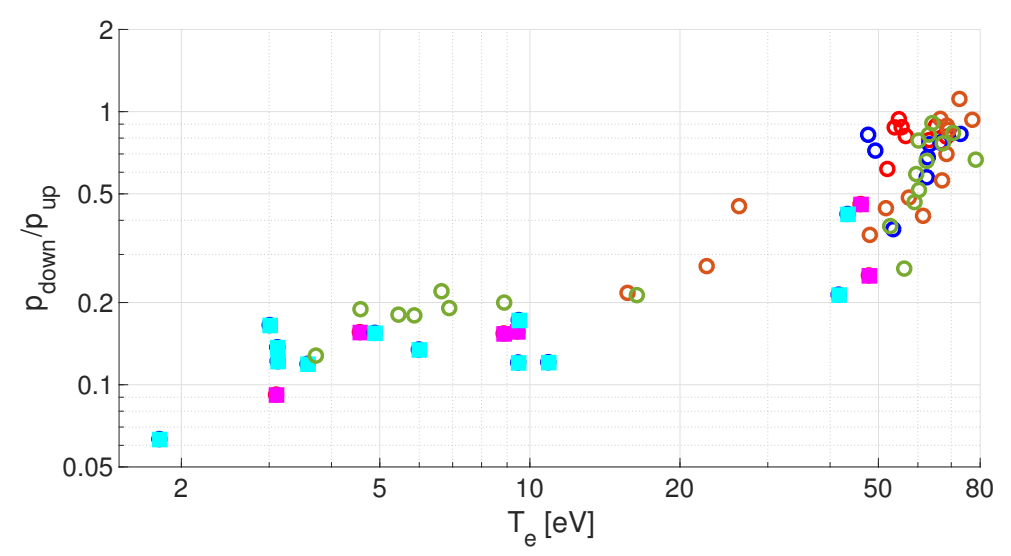

Figure 15. Ratio of downstream $\left(p_{\text {down }}\right)$ and upstream $\left(p_{u p}\right)$ pressures as a function of maximum $T_{e}$ at the outer target in different discharges with LFS nitrogen seeding. Square symbols indicate application of NBI.

confined region.

In order to resolve this issue, a new set of experiments was performed, where the effect of nitrogen radiation inside the separatrix was compensated by the application of NBI heating. The effect of auxiliary heating on the upstream profiles was expected to be only moderate, so maximum available power of $450 \mathrm{~kW}$ was delivered into the plasma at $\mathrm{t}=1120 \mathrm{~ms}$ (the beginning of the seeding waveform was shifted to $t=1080 \mathrm{~ms}$ in these discharges). Note that in a non-seeded discharge this would lead to access to H-mode but the presence of nitrogen clearly prevents it.

The achieved pressure drop is summarised in Fig. 15, where the ratio of the downstream and upstream pressures in all discharges with LFS nitrogen seeding is plotted against the target temperature. The lowest pressure fractions were achieved during the NBI phases (marked by squares in the figure) and represent a clear demonstration of partial detachment. Note that the shape of the dependency on $T_{e}$ is quite different than observed at C-mod[21] and AUG [22]. This can be a consequence of the radiation of nitrogen in the confined plasma, which is not so detrimental in larger devices.

\section{Buffered heat flux at the outer target}

The principal objective of operation in detached regime is to mitigate heat fluxes impinging onto the divertor targets, in order to avoid damage of the plasma-facing components. This is needed already in some of the contemporary machines (such as AUG) in high-power scenarios and it will be unavoidable for ITER and next-step machines. In order to evaluate the effect of impurity seeding on the heat fluxes, radial profiles of heat flux were constructed based on probe measurements at the outer target using Eq. 4.

Figure 16 summarises the heat flux measurements, with data from discharges, where nitrogen was introduced at the LFS. The impurity injection is causing large decrease of the heat flux close to the strike point (marked by vertical dashed line), reducing it more than factor 

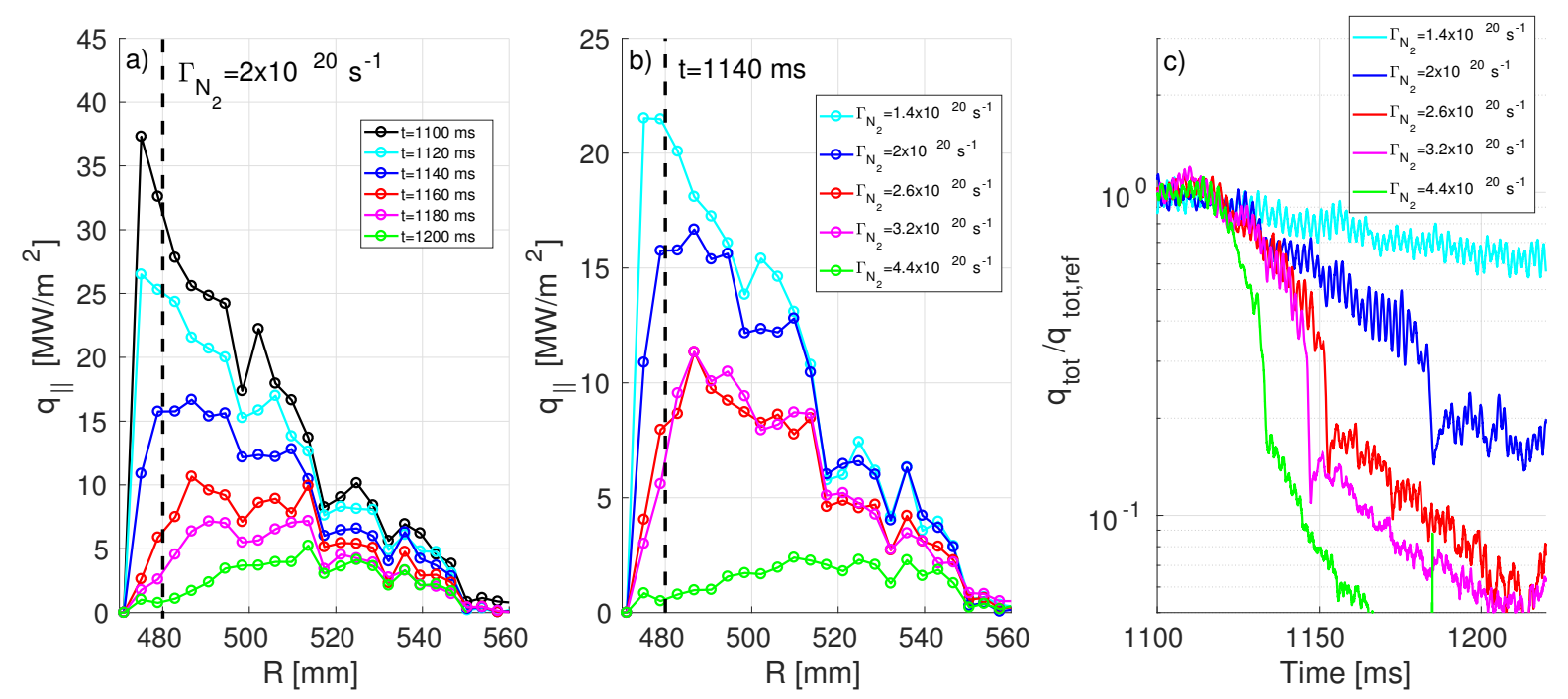

Figure 16. Temporal evolution of heat flux during a seeding experiment in the vicinity of the outer strike point (a), Heat flux profiles at $\mathrm{t}=1140 \mathrm{~ms}$ for varying amount of seeding (b) and ratio of integral heat flux at the outer target $q_{t o t}$ normalized to pre-seeding level $q_{t o t, r e f}$ (c).

of 10 , while further away the reduction is less significant.

Instead of attempting to characterise directly the heat flux footprints during the nitrogen seeding, one can use the attached heat flux profile as a reference $q_{r e f}$ (black line in Fig.16A) and introduce a buffered heat flux $q_{B}$, the heat flux which has been removed from the footprint due to the presence of injected impurity:

$$
q_{B}(t, R)=\left(q_{r e f}(R)-q(t, R)\right) / q_{r e f}(R) .
$$

Since the effect of the nitrogen seeding is believed to be localised in the divertor region, we prefer not to map the profiles to the outer midplane but instead use the radial coordinate $R$ at the target. An example of the profiles of buffered flux $q_{B}$ in case of moderate seeding at the LFS are shown in Fig. 17A. The profiles can be well characterised by an exponential decay

$$
q_{B}(R)=A_{B} \exp \left(-\left(R-R_{O S P}\right) / \lambda_{B}\right)
$$

Due to the limited data set it is difficult to determine if exponential dependency is the best fit of the decay, neither it is backed by any hypothesis on underlying physical processes. However, it allows to characterise the main features of the buffering: the magnitude of buffering at the strike point $A_{B}$ and the spatial extend of the buffering $\lambda_{B}$. Note that the values of $\lambda_{B}$ are significantly larger than the $\lambda_{q}$ in the attached plasmas (typically between $20-40 \mathrm{~mm}$ at outer target).

These parameters are expected to be primarily dependent on the inventory of nitrogen in the plasma and consequent radiation losses. Indeed, both parameters exhibit strong dependencies on $f_{\text {rad }}$, as shown in Fig. 18. While the evolution of the parameter $A_{B}$ can be characterised by a linear increase for $0.1<f_{\text {rad }}<0.2, \lambda_{B}$ continues to increase further also for higher values of $f_{\text {rad }}$ with an approximately exponential dependence. Only fits with 

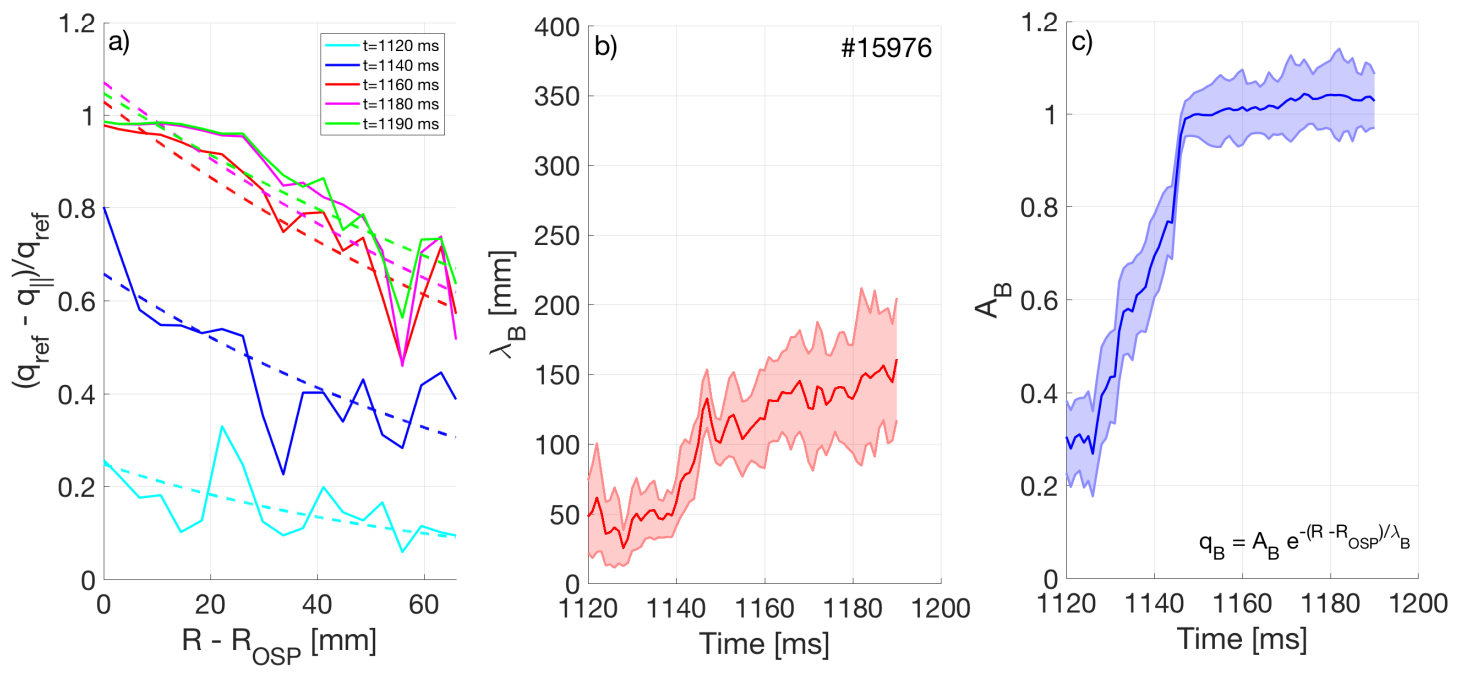

Figure 17. Buffered heat flux during discharge \#15976 (A) and the time evolution of the fitting parameters $\lambda_{B}$ (B) and $A_{B}(\mathrm{C})$.
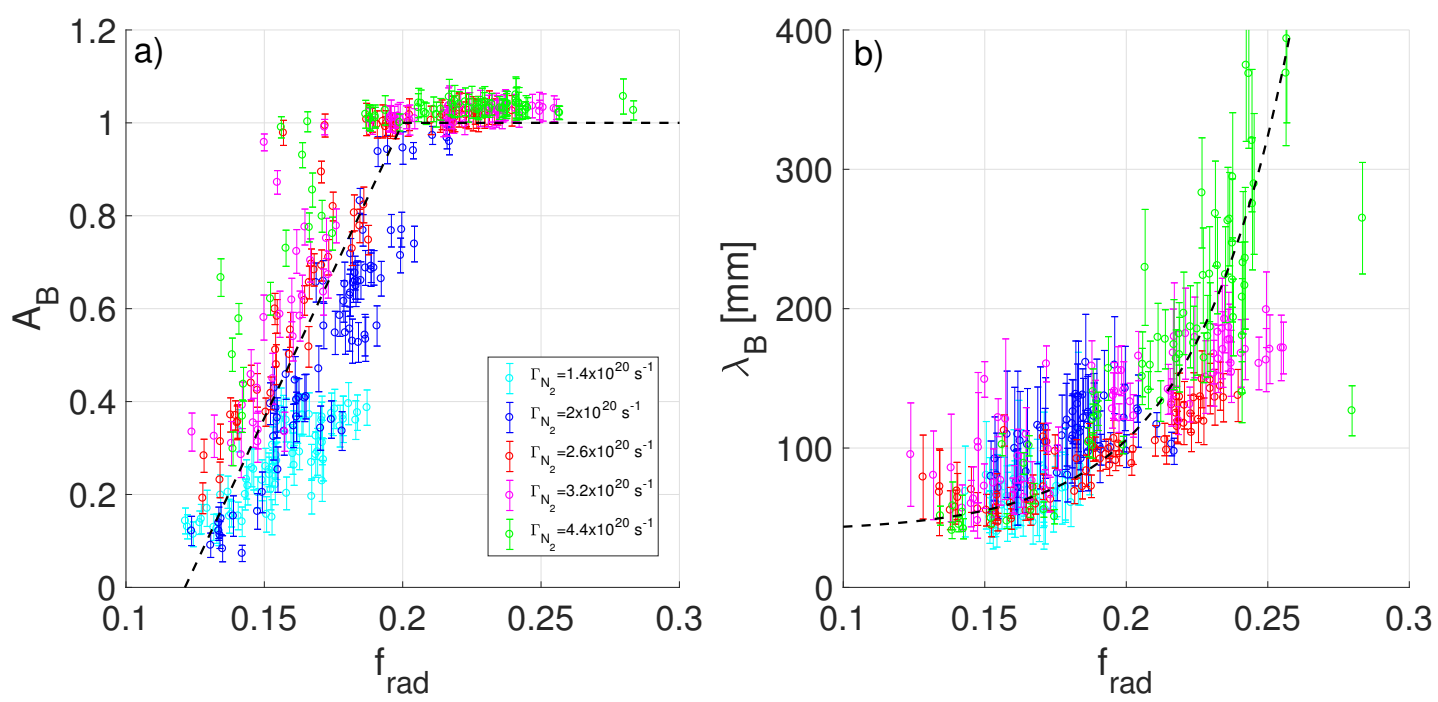

Figure 18. Dependence of buffer fit parameters $A_{B}$ (left) and $\lambda_{B}$ (right) on the radiated fraction in the discharge.

error lower than $50 \%$ were used for this analysis, which discarded in particular a number of fits at low $f_{\text {rad }}$. In such cases the buffering of heat fluxes is small and so the $q_{B}$ is subject to higher level of fluctuations.

\section{Characterisation of the partially detached divertor footprints}

The divertor heat fluxes in attached conditions are typically mapped to outer midplane and characterised by a function proposed by Eich in Ref. [23], which is a convolution of an exponential decay (with power decay length $\lambda_{q}$ ) and the Gaussian broadening of the profile 

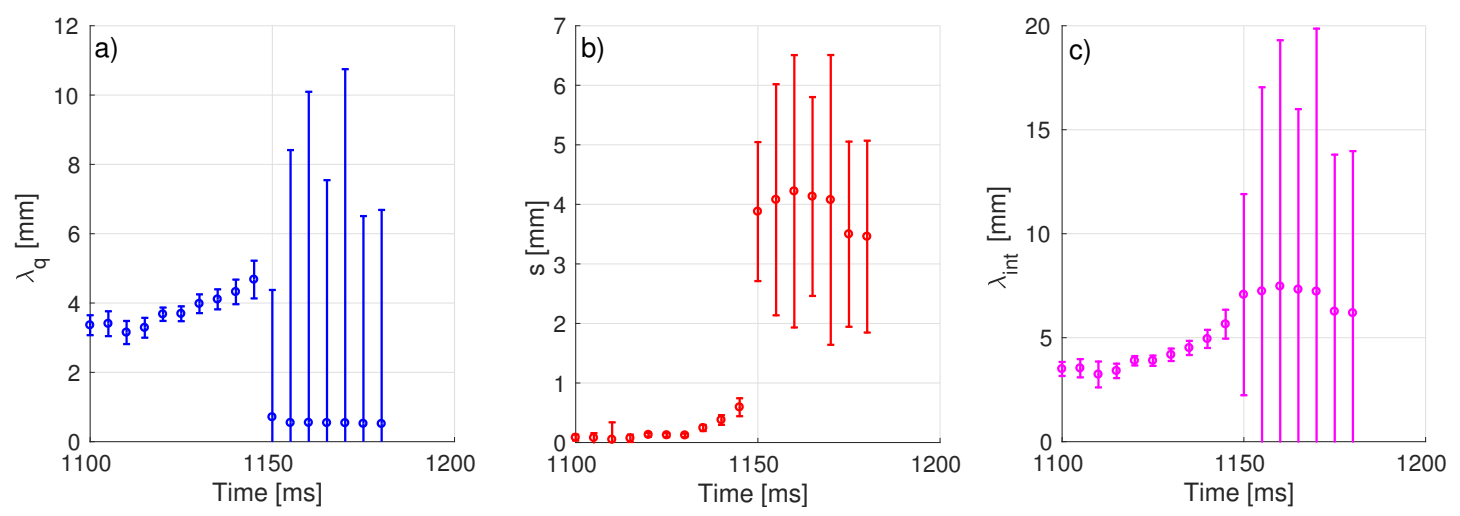

Figure 19. Time evolution of $\lambda_{q}(\mathrm{~A}), s(\mathrm{~B})$ and $\lambda_{\text {int }}(\mathrm{C})$ in shot \#15976

(with characteristic width $S$ ) given by

$$
q_{s}=\frac{q_{0}}{2} \exp \left(\left(\frac{S}{2 \lambda_{q}}\right)^{2}-\frac{s}{\lambda_{q} f_{x}}\right) \cdot \operatorname{erfc}\left(\frac{S}{2 \lambda_{q}}-\frac{s}{S f_{x}}\right)+q_{B G},
$$

where $f_{x}$ is the flux expansion at the target, $q_{0}$ the peak heat flux, $q_{B G}$ the background heat flux due to radiation and $s$ is the spatial coordinate running along the target in poloidal direction. This function reflects the assumption that the divertor target profile is partially determined by upstream conditions (exponential decay) and partially by processes in the divertor region (collisions and finite Larmor effects [13]). With respect to the spatial extend of the power deposition, the footprint can be approximated by an exponential decay with characteristic integral power decay length $\lambda_{\text {int }}$ [24]

$$
\lambda_{\text {int }}=\lambda_{q}+1.64 S \text {. }
$$

This characterisation proved to be successful in accurate description of heat flux profiles on a number of machines [23]. However, its domain of applicability is limited by the physical picture described above, which does not include e.g. dissipative processes, where part of the power is converted into radiation. With 5 degrees of freedom available, the formula 7 can be still technically applied to fit the target profiles even when such processes are significant (as it is the case in detached plasmas) but in such case the fitting parameters $\lambda_{q}$ and $S$ are losing their original meaning. In order to illustrate it, we have analyzed discharge \#15976 $\left(\Gamma_{N_{2}}=4.4 \times 10^{20} \mathrm{~s}^{-1}\right)$ and plotted the time evolution of $\lambda_{q}, S$ and $\lambda_{\text {int }}$ in Fig. 19. It can be seen that the fitted $\lambda_{q}$ is increasing during the initial part of the seeding until the transition to detachment (around $\mathrm{t}=1150 \mathrm{~ms}$ ), when it sharply drops and the quality of the fit significantly decreases. However, the main parameters, which determine it according to L-mode multimachine scaling by Eich[34] $\left(B_{T}\right.$ and $\left.q_{c y l}\right)$, remain constant during the flat-top. The apparent change of $\lambda_{q}$ is a consequence of different physical processes influencing the divertor footprint - the effect of nitrogen seeding has different nature than a Gaussian broadening of the profile.

In order to analyse the profiles in detached plasmas (as shown in Fig. 16A,B), a new set of parameters should be implemented to allow multi-machine comparisons. The approach presented in the previous section is feared to be limited to particular conditions 

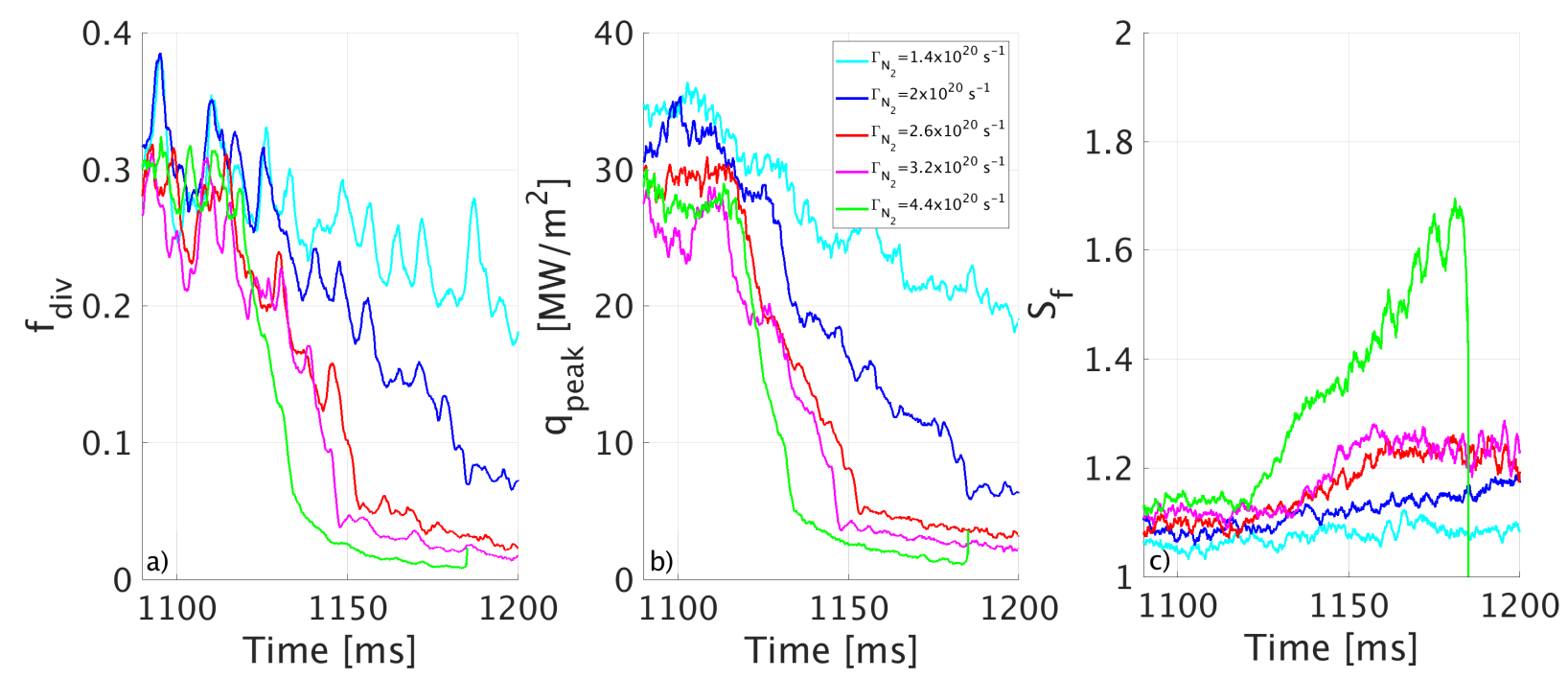

Figure 20. Time evolution of $f_{d i v}$ (A), $q_{p e a k}(\mathrm{~B})$ and $S_{f}(\mathrm{C})$ in discharges with LFS nitrogen seeding

of the COMPASS divertor and may not be applicable on other tokamaks. Since the problem of heat fluxes is mostly an engineering issue, we propose to charaterise the heat fluxes by a set of parameters, which are suitable for evaluation of safety issues related to PFCs. These are the following: (i) peak heat flux $q_{\text {peak }}$ being the maximum of heat flux (from which the background radiation $q_{B G}$ is substracted), (ii) divertor power fraction $f_{d i v}=2 \pi R \int q_{\|}(s) \sin (\alpha(s)) d s / P_{\text {sep }}$ being the fraction of power crossing the separatrix which reaches the target and (iii) footprint spreading factor $S_{f}$ - minimal distance along the target over which $1-1 / e \sim 63 \%$ of the power is deposited, normalized by the expected footprint width in an idealized case of attached plasmas in absence of spreading ( $\mathrm{S}=0) \lambda_{q} f_{x}$.

In case of attached plasmas with constant angle of incidence $\alpha$, one can relate the new set of parameters with those implemented by Eich:

$$
q_{\text {peak }}=q_{0} \frac{\lambda_{q}}{\lambda_{\text {int }}} ; f_{\text {div }}=2 \pi R f_{x} q_{0} \lambda_{q} \sin (\alpha) / P_{\text {sep }} ; S_{f}=\lambda_{\text {int }} / \lambda_{q}=1+1.64 S / \lambda_{q}(9)
$$

Time traces shown in Fig. 20 show evolution of the new set of parameters obtained at the outer target. It can be seen that while $f_{\text {div }}$ and $q_{\text {peak }}$ are changing dramatically during the impurity seeding, the third parameter $S_{f}$ shows significant increase only for the highest seeding rate.

As with the parameters describing the buffered heat flux, it is again expected that the main factor influencing their evolution is the nitrogen radiation in the plasma. The dependencies (shown in Fig. 21) confirm this expectation. Both $q_{p e a k}$ and $f_{d i v}$ are steadily decreasing as $f_{\text {rad }}$ exceeds a threshold value 0.15 , with an approximately exponential dependence. 

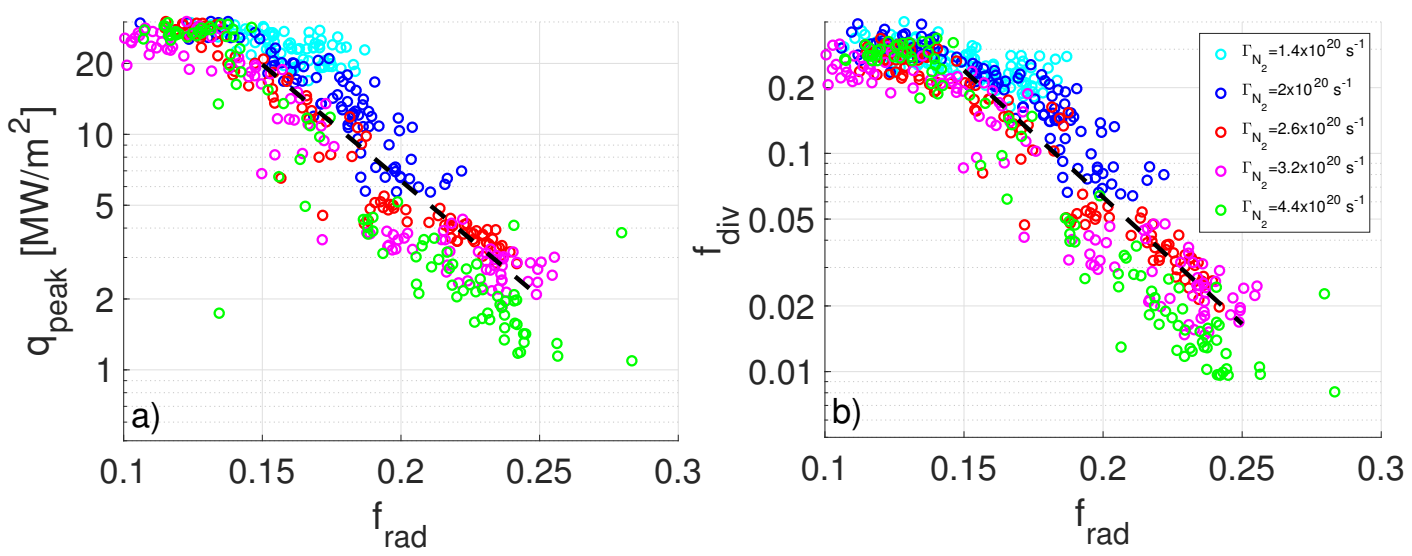

Figure 21. Dependence of parameters $q_{p e a k}$ and $f_{d i v}$ on the radiated fraction $f_{\text {rad }}$

\section{Comparison with the detachment scaling model}

A recent model for detachment scaling introduced by Goldston [26] allows to predict the nitrogen concentration required for detachment. In order to compare COMPASS data with the results presented in [26], it is necessary to account for the fact that our experiments were performed in L-mode, where the power decay length is expected to be larger than in H-mode. Indeed, the heuristic drift model [27] predicts $\lambda_{q, H D}=2.5 \mathrm{~mm}$, while analysis of the footprint at outer target prior to seeding show $\lambda_{q}=4 \mathrm{~mm}$. In order to rescale the results obtained for AUG in [26], we need to calculate the factor $P_{\text {sep }} /\left(\left(\lambda_{q} / \lambda_{q, H D}\right)^{8 / 7}<B_{p}>\left(n_{\text {sep }} / n_{\text {gw }}\right)^{2} l_{\|}^{*}\right)$, which has a value of $200\left(P_{\text {sep }}=230 \mathrm{~kW},\left\langle B_{p}>=0.16 \mathrm{~T}, n_{\text {sep }}=1.7 \times 10^{19} \mathrm{~m}^{-3}, n_{\text {gw }}=\right.\right.$ $\left.21.8 \times 10^{19} \mathrm{~m}^{-3}, L_{c o n}=3.9 \mathrm{~m}, q_{c y l}=4.2, l_{\|}^{*}=0.54\right)$. Using data from Table 2 in [26], one finds the predicted impurity fraction $c_{z, \text { Goldston }}=7.6 \%$, which is higher than on JET or AUG but lower than the fraction predicted for ITER.

Unfortunately, the analysis of spectroscopy data does not allow precise confrontation of this prediction. The estimation based on spectral measurements presented in section 3.2 allows only for rough comparison, since the key parameters $\tau$ and $\Delta L$ are not known with sufficient precision. Another rough estimate of nitrogen concentration can be obtained from the knowledge of nitrogen seeding rate, which can deliver $1-4 \times 10^{19}$ particles into the vessel during the discharge. Given the approximate vessel volume of $1 \mathrm{~m}^{3}$ and the line-averaged density of $4.0 \times 10^{19} \mathrm{~m}^{-3}$, the $c_{z}$ would be $25-100 \%$ if nitrogen would be distributed uniformly and in the absence of nitrogen removal mechanisms. This is clearly too high for the plasma to sustain operation.

The most realistic estimate can be obtained from calculations of the cooling power $P_{\text {cool }}=c_{z} n_{e}^{2} L_{z}$, which as seen in Fig. 16 can compensate almost the whole $P_{\text {sep }}$. The radiative loss function $L_{z}$ can be approximately determined from Fig. 3 in [28] as $2 \times 10^{-32}$ $\mathrm{Wm}^{3}$, which yields $c_{z}$ of 5 percent, a value consistent with Goldston's prediction. 


\section{Conclusions}

Measurements of plasma parameters in COMPASS tokamak during nitrogen seeding were performed using a variety of diagnostics. The most important tool for the evaluation of plasma conditions in the divertor region was the combined array of Ball-pen and Langmuir probes. Despite persisting open questions in the evaluation of probe data in detached conditions, relative changes of electron pressure and heat flux allowed to characterise the effects of nitrogen seeding.

Nitrogen seeding was proved to be an efficient tool for reduction of divertor pressure and heat flux in a series of dedicated L-mode discharges at COMPASS. The plasma response to the seeding is in general dependent on the location of the seeding, with more favourable results being achieved with seeding in the vicinity of the outer strike point. The nitrogen radiation is not restricted to the divertor region but affects also the confined plasma, which results in considerable upstream temperature drop. During the NBI-assisted discharges the ratio of upstream and downstream pressure changed by a factor of 10 , which is a clear demonstration of partial detachment.

In order to characterise the effect of nitrogen seeding on the divertor footprint, the buffered heat flux $q_{B}$ was introduced. Buffered heat flux at COMPASS can be well approximated by an exponential decay with large characteristic length. Both parameters describing $q_{B}$ exhibit a strong dependence on radiated fraction, however this feature remains to be tested on data from other machines. Also, a more generic way of characterising the heat fluxes was proposed, to answer the practical questions related to the engineering limits of the plasma-facing components. The peak heat flux $q_{\text {peak }}$ and divertor power fraction $f_{d i v}$ again depend strongly on the radiated fraction, however the footprint spreading factor $S_{f}$ is insensitive and remains constant during the seeding in most of the discharges.

\section{Acknowledgments}

First author would like to thank R. J. Goldston for valuable discussion related to his detachment scaling model and T. Eich for discussion on the $\lambda_{q}$ scaling. This work has been carried out within the framework of the EUROFusion Consortium and has received funding from the Euratom research and training programme 2014-2018 and 2019-2020 under grant agreement No 633053. The views and opinions expressed herein do not necessarily reflect those of the European Commission. It was supported by projects Czech Science Foundation GA15-10723S, GA16-14228S, MYES projects \#LM2015045 and CZ.02.1.01/0.0/0.0/16_013/0001551 and IAEA CRP F13019 - Research Contract No. $22727 / \mathrm{R} 0$.

[1] M. Komm et al., proceedings of the $44^{\text {th }}$ EPS confererence (2017) P1.118

[2] J. Adamek et al. Nucl. Fusion 57 (2017) 116017

[3] J. Adamek et al. Rev. Sci. Instr. 87 (2016) 043510

[4] D. Brunner et al. Nucl. Fusion 58 (2016) 094002

[5] O. Fevrier et al. Rev. Sci. Instrum. 89 (2018) 053502

[6] N. Ohno et al. Contrib. Plasma Phys. 41 (2001) 473-480 
[7] J. Mlynar et al., Rev. Sci. Instr. 83 10E531 (2012)

[8] A. Havranek et al., Fusion Eng. and Design 123 (2017) 857-860

[9] M. Aftanas et al., Rev. Sci. Instr. 83 (2012) 10E350

[10] T. Eich et al. Nucl. Fusion 58 (2018) 034001

[11] P. C Stangeby The plasma boundary of magnetic fusion devices IOP Publishing (2000)

[12] Yu. Igitkhanov and G. Janeschitz J. Nucl. Mater. 99 (2001) 290-293

[13] B. Sieglin et al. Plasma Phys. Control. Fusion 58 (2016) 055015

[14] J. P. Gunn et al. Nucl. Fusion 57 (2017) 046025

[15] A.S. Kukushkin et al. Nucl. Fusion 49 (2009) 075008

[16] T.W. Petrie et al. J. Nucl. Mat. 241-243 (1997) 639-644

[17] A. Podolnik et al. Plasma Phys. Control. Fusion 60 (2018) 085008

[18] K. Jirakova et al. Proceedings of the 45th EPS conference, Prague (2018) P5.1081

[19] J. Adamek et al. Nucl. Fusion 57 (2017) 022010

[20] A.G. McLean et al. J. Nucl. Mat. 463 (2015) 533-536

[21] B. Lipschultz et al., Fusion Sci. Tech. 51 (2017) 369-389

[22] I.P. Perez et al. Nucl. Mat. Energy 12 (2017) 182-186

[23] T. Eich et al., Nucl. Fusion 53 (2013) 093031

[24] M.A. Makowski Phys. Plasmas 19 (2012) 056122

[25] D. Naydenkova et al. Appl. Optics 53 (2014) 8123-8130

[26] R. J. Goldston et al. Plasma Phys. Control. Fusion 59 (2017) 055015

[27] R. J. Goldston et al. Nucl. Fusion 52 (2012) 013009

[28] A. Kallenbach et al. Plasma Phys. Control. Fusion 58 (2016) 045013

[29] A. Scarabosio et al. J. Nucl. Mater. 438 (2013) S426S430

[30] F. Reimold et al. J. Nucl. Fusion 55 (2015) 033004

[31] A. Loarte Plasma Phys. Control. Fusion 43 (2001) R183-R224

[32] A. Kallenbach et al., Nucl. Fusion 57 (2017) 102015

[33] S. S. Henderson et al., Nucl. Fusion 58 (2018) 016047

[34] T. Eich et al., J. Nucl. Mat.438 (2013) S72-S7

[35] J. Seidl et al., Nucl. Fusion 57 (2017) 126048

[36] A. V. Melnikov et al., Plasma Phys. Control Fusion 57 (2015) 065006

[37] T. Markovic et al., Proceedings of the 44th EPS conference (2017) P5.140

[38] F. Reimold et al. Nucl. Fusion 55 (2015) 033004

[39] S. Potzel et al. Nucl. Fusion 54 (2014) 013001

[40] S. Potzel et al. J. Nucl. Mat. 438 (2013) S285-S290

[41] A. Stepanenko et al. Phys. of Plasmas 25 (2018) 012305

[42] S. I. Krasheninnikov et al. Nucl. Mat. Energy 12 (2017) 1061-1066

[43] S. I. Krasheninnikov et al. Phys. Plasmas 23 (2016) 092505

[44] P. Vondracek, Plasma heat flux to solid structures in tokamaks, dissertation thesis https://is.cuni.cz/webapps/zzp/detail/123000/.

[45] M. Komm et al., Plasma Phys. Control. Fusion 59 (2017) 094002

[46] C.S. Silva, J. Nucl. Mat. 266-269 (1999) 679-684 